\title{
OBSTRUCTION THEORY OF PRINCIPAL FIBRE BUNDLES
}

\author{
BY \\ ALBERT T. LUNDELL( $(1)$
}

Introduction. The object of this paper is the computation of higher obstructions to cross-sections of a principal fibre bundle $\xi=(E, p, B, G, G)$, in terms of the Postnikov invariants of the fibre. This calculation can be carried out explicitly in the case where $G$ is a connected countable CW-group and $B$ is a locally finite simplicial polyhedron, modulo the usual difficulties in computing the Postnikov invariants.

In a research announcement, Hermann [9] describes a method for computing higher obstructions to a cross-section for a general fibre space $(E, p, B, F)$, in which he makes use of the Moore-Postnikov decomposition of the fibre space. For a countable $\mathrm{CW}$-group $G$, there is a (finite) Postnikov system in which each space is a countable $\mathrm{CW}$-group and the maps are continuous homomorphisms. This fact leads to a construction which is "dual" to that of Hermann in the sense that certain fibre maps in Hermann's construction are replaced by inclusion maps. The fibre spaces in this decomposition are all principal fibre bundles with the terms of the Postnikov system of $G$ as the fibre. It is due to the additional structure of a principal fibre space that our computation is feasible. While Hermann's method requires that $G$ have relatively "nice" homotopy groups for effective computation, the method we describe works for any (finitely generated) homotopy groups. An example at the end of $\S 6$, in which we consider a principal $S^{3}$-bundle, illustrates the difference between Hermann's work and ours. We suspect that the simpler formulae obtained by our method in this case is typical of the difference which would result on application of the two methods to an arbitrary principal bundle.

The first five sections of this paper give preliminary results and constructions, and the main results occur in $\$ 6$. In the last three sections, we give applications to bundles whose fibre is $S O(n), U(n)$, and $S p(n)$, and in the case of $S O(n), n \geqq 5$, and $U(n)$, we are able to calculate the second and third obstructions. In some special cases, we are able to relate these higher obstructions to certain characteristic classes of the bundle.

The author wishes to acknowledge his indebtedness to Professor W. S. Massey for many valuable discussions during the preparation of this paper.

1. Definitions and conventions. A fibre space is an ordered quadruple $\xi=(E, p, B, F)$, where $E, B$, and $F$ are topological spaces and $p: E \rightarrow B$ is a

Received by the editors February 10, 1960.

(1) The author was partially supported by the National Science Foundation during the preparation of this paper. 
continuous function, such that the following "local triviality" condition holds: for each point $x \in B$ there is a neighborhood $U$ of $x$ and a homeomorphism $\phi_{U}$ of $U \times F$ on $p^{-1}(U)$ with the property that $p \phi_{U}(y, z)=y$ for $(y, z)$ $\in U \times F . E, B$, and $F$ are the total space, base, and fibre, respectively, and $p$ is the projection of $\xi=(E, p, B, F)$. A principal fibre space $\xi=(E, p, B, G)$ is a fibre space where the fibre $G$ is a topological group and for which there exists a continuous function $\mu: G \times E \rightarrow E$ (we denote $\mu(g, x)$ by $g \cdot x$ ) such that if $\phi_{U}: U \times G \rightarrow p^{-1}(U)$ is a homeomorphism defining local triviality as above, if $b \in U$ and $g, g^{\prime} \in G$, then $g^{\prime} \cdot \phi_{U}(b, g)=\phi_{U}\left(b, g^{\prime} \cdot g\right)$, and such that

(i) $g \cdot x=x$ if and only if $g=\dot{e}$, the identity of $G$ for $x \in E$,

(ii) $g^{\prime} \cdot(g \cdot x)=\left(g^{\prime} \cdot g\right) \cdot x$ for $g, g^{\prime} \in G$ and $x \in E$.

A cross-section of a fibre space $\xi=(E, p, B, F)$ is a continuous function $f: B \rightarrow E$ such that $p f(b)=b$ for all $b \in B$. Two cross-sections $f_{0}, f_{1}: B \rightarrow E$ are homotopic if and only if there is a continuous function $h: B \times I \rightarrow E$ such that $h(b, 0)=f_{0}(b), h(b, 1)=f_{1}(b)$, and $p h(b, t)=b$ for $b \in B$ and $0 \leqq t \leqq 1$.

If $\xi=(E, p, B, F)$ is a fibre space and $\phi: B^{\prime} \rightarrow B$ is a map, then $\phi^{-1}(\xi)$ will denote the induced fibre space [6, Exposé 6].

Throughout this paper, we will assume that the base space $B$ is a locally finite connected simplicial polyhedron. $B^{n}$ will denote the $n$-skeleton of $B$. We will also assume that the fibre $F$ is connected and is $n$-simple in all dimensions.

We will use the singular homology theory, and if no coefficients are mentioned, we will assume they are in the integers. The group of integers will be denoted by $Z$, and the group of integers $(\bmod n)$ by $Z_{n}$.

2. Fibre subspaces. Let $\xi=(E, p, B, F)$ and $\xi^{\prime}=\left(E^{\prime}, p^{\prime}, B, F^{\prime}\right)$ be fibre spaces over the same base $B$. We say that $\xi^{\prime}$ is a fibre subspace of $\xi$ provided $E^{\prime} \subset E$, the inclusion $j: E^{\prime} \rightarrow E$ satisfies $p j=p^{\prime}$, and for each $x \in B$ there is a neighborhood $U$ of $x$ and a homeomorphism of pairs $\phi_{U}:\left(U \times F, U \times F^{\prime}\right)$ $\rightarrow\left(p^{-1}(U), p^{\prime-1}(U)\right)$ such that $p \phi_{U}(y, z)=y$ for $(y, z) \in U \times F$. A pair of fibre spaces $\left(\xi, \xi^{\prime}\right)$ will consist of a fibre space $\xi$ and a fibre subspace $\xi^{\prime}$. If $\left(\xi, \xi^{\prime}\right)$ is a pair over the base $B$, and if $\phi: A \rightarrow B$ is a map, then $\left(\phi^{-1}(\xi), \phi^{-1}\left(\xi^{\prime}\right)\right)$ is a pair over $A$ called the induced pair, and will be denoted by $\phi^{-1}\left(\xi, \xi^{\prime}\right)$. If $\phi$ is an inclusion map, we will write $\left(\xi, \xi^{\prime}\right)_{A}=\left(\xi_{A}, \xi_{A}^{\prime}\right)$. For notational purposes, $\xi_{A}=\left(E_{A}, p_{A}, A, F\right)$ and $\xi_{A}^{\prime}=\left(E_{A}^{\prime}, p_{A}^{\prime}, A, F^{\prime}\right)$. This notation holds throughout this section.

We will need the following form of the covering homotopy theorem.

TheOREm 2.1. Let $\xi=(E, p, B, F)$ be a fibre space, let $X$ be locally compact and paracompact, and let $A$ be a closed subset of $X$. Suppose $f: X \rightarrow E$ and $h: X \times I \rightarrow B$ are such that $p f=h \mid X \times\{0\}$. Suppose further that there is a "partial lifting" of $h$ on $A$, i.e., a map $h^{\prime}: A \times I \rightarrow E$ such that $p h^{\prime}=h \mid A \times I$ and $h^{\prime}|A \times\{0\}=f| A$. Then there exists a map $h: X \times I \rightarrow E$ such that $p h=h$, $\tilde{h} \mid X \times\{0\}=f$ and $\tilde{h} \mid A \times I=h^{\prime}$. 
The proof requires a trivial modification of the proof of the covering homotopy theorem given by Cartan [6, Exposé 8]. We suspect, but have not proved, that this theorem holds without the assumption of local compactness.

The following corollary gives a covering homotopy theorem for pairs of fibre spaces.

CoRollaRy 2.2. Let $\left(\xi, \xi^{\prime}\right)$ be a pair of fibre spaces over the base space $B$, let $X$ be locally compact and paracompact, and let $A$ be closed in $X$. Suppose $f:(X, A) \rightarrow\left(E, E^{\prime}\right)$ and $h: X \times I \rightarrow B$ are maps such that $p f=h \mid X \times\{0\}$. Then there exists a map $\bar{h}:(X \times I, A \times I) \rightarrow\left(E, E^{\prime}\right)$ such that $\bar{h} \mid X \times\{0\}=f$ and $p h=h$.

Proof. First apply the usual covering homotopy theorem in $\xi^{\prime}$ to obtain $h^{\prime}: A \times I \rightarrow E^{\prime}$ such that $p h^{\prime}=h \mid A \times I$ and $h^{\prime}|A \times\{0\}=f| A$. An application of Theorem 2.1 completes the proof.

The propositions of this section have to do with homotopy and homology properties of a pair of fibre spaces, which will be used in our theory of obstructions to cross-sections and in some applications of this theory.

LemMA 2.3. Let $\left(\xi, \xi^{\prime}\right)$ be a pair of fibre spaces, and let $j: E \rightarrow E^{\prime}$ be the inclusion map. Let $A \subset B$ with inclusion $k: A \rightarrow B$, and let $\left(\xi_{A}, \xi_{A}^{\prime}\right)$ be the induced pair. Then for each integer $m \geqq 2$, the inclusion $\bar{k}:\left(E_{A}, E_{A}^{\prime}\right) \rightarrow\left(E, E^{\prime}\right)$ induces an isomorphism onto

$$
\bar{k}_{*}: \pi_{m}\left(E_{A}, E_{A}{ }^{\prime}\right) \stackrel{\approx}{\rightarrow} \pi_{m}\left(E, E^{\prime}\right),
$$

and there is a direct sum decomposition $\pi_{m}\left(E, E_{\Lambda}^{\prime}\right) \approx \pi_{m}\left(E, E^{\prime}\right)+\pi_{m}(B, A)$.

Proof. Consider the commutative diagram:

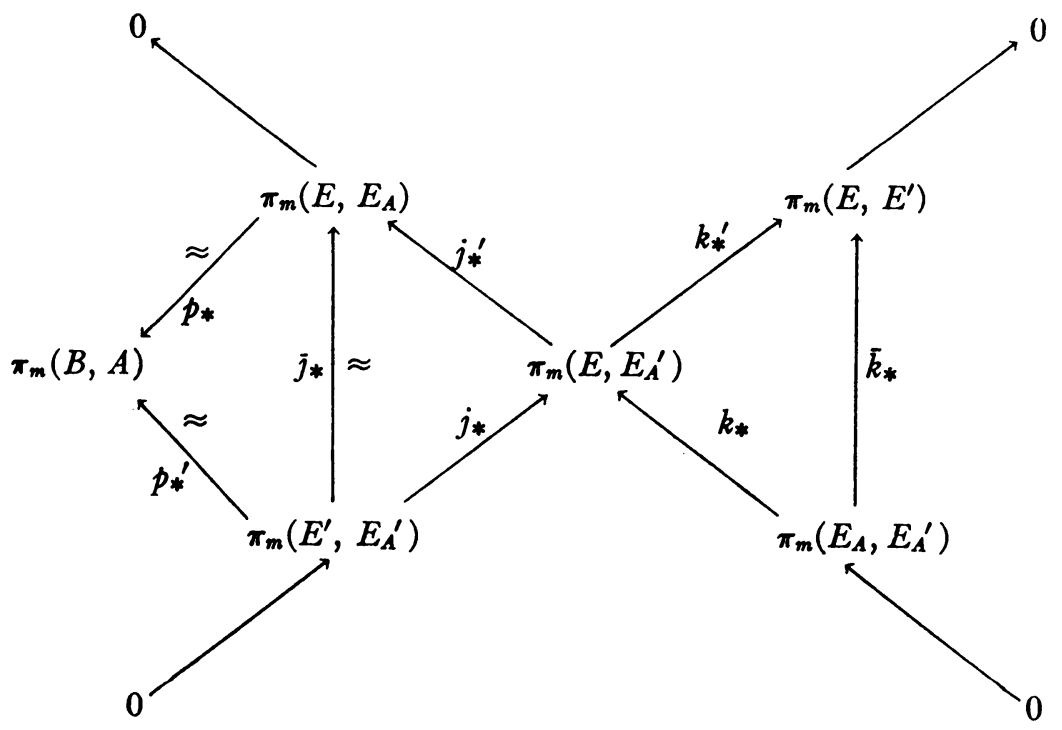


where the diagonal sequences are portions of the exact homotopy sequences of the triples $\left(E, E^{\prime}, E_{A}^{\prime}\right)$ and $\left(E, E_{A}, E_{A}^{\prime}\right)$. Since $p j=p^{\prime}$ and $p_{*}$ and $p_{*}^{\prime}$ are isomorphisms onto, these sequences break up into sequences of length three and $\bar{j}_{*}$ is an isomorphism onto, as shown in the diagram.

If $x \in \pi_{m}\left(E_{A}, E_{A}^{\prime}\right)$ and $0=k_{*}(x)=k_{*}^{\prime} k_{*}(x)$, then $k_{*}(x)=j_{*}(y)$, and $\bar{j}_{*}(y)$ $=j_{*} j_{*}^{\prime}(y)=j_{*}^{\prime} k_{*}(x)=0$, so $y=0$. But then $k_{*}(x)=0$, which implies $\bar{k}_{*}$ is oneto-one, because $k_{*}$ is one-to-one.

If $x \in \pi_{m}\left(E, E^{\prime}\right)$, then $x=k_{*}^{\prime}(y)$. Since $j_{*}^{\prime}(y)=j_{*}(z)=j_{*}^{\prime} j_{*}(z), y-j_{*}(z)$ $=k_{*}(w)$. But $\bar{k}_{*}(w)=k_{*}^{\prime} k_{*}(w)=k_{*}^{\prime}\left(y-j_{*}(z)\right)=k_{*}^{\prime}(y)=x$, and $\bar{k}_{*}$ is onto.

The statement about the direct sum decomposition follows from a lemma of Eilenberg and Steenrod [8, p. 32].

COROLlaRY 2.4. If $\left(\xi, \xi^{\prime}\right)$ is a pair of fibre spaces, then for each integer $m \geqq 2$, the inclusion $i:\left(F, F^{\prime}\right) \rightarrow\left(E, E^{\prime}\right)$ induces an isomorphism onto

$$
\bar{i}_{*}: \pi_{m}\left(F, F^{\prime}\right) \stackrel{\approx}{\rightarrow} \pi_{m}\left(E, E^{\prime}\right) .
$$

Proof Take $A=\{x\}$ for $x \in B$, and apply the lemma.

Let $\left(\xi, \xi^{\prime}\right)$ be a pair of fibre spaces such that $\left(F, F^{\prime}\right)$ is $m$-simple, i.e., $\pi_{1}\left(F^{\prime}\right)$ operates trivially on $\pi_{m}\left(F, F^{\prime}\right)$. Then by the use of Corollary 2.2, we are able to define operations of $\pi_{1}(B)$ on $\pi_{m}\left(F, F^{\prime}\right)$ entirely analogous to the usual operations of $\pi_{1}(B)$ on the homotopy groups of the fibre induced by "translating the fibre around a curve." Specifically, let $\alpha:\left(\sigma^{m}, \dot{\sigma}^{m}, z_{0}\right)$ $\rightarrow\left(F, F^{\prime}, y_{0}\right)$ be a mapping of the oriented $m$-cell which represents the element $\bar{\alpha} \in \pi_{m}\left(F, F^{\prime}, y_{0}\right)$, let $\xi_{b_{0}}:\left(F, F^{\prime}\right) \rightarrow\left(E, E^{\prime}\right)$ be an admissible map, let $x_{0}=\xi_{b_{0}}\left(y_{0}\right)$, and let $\rho:\left(\sigma^{1}, \dot{\sigma}^{1}\right) \rightarrow\left(B, b_{0}\right)$ be a map representing $\bar{\rho} \in \pi_{1}\left(B, b_{0}\right)$. Then $\xi_{b_{0}} \alpha:\left(\sigma^{m}, \dot{\sigma}^{m}, z_{0}\right) \rightarrow\left(E, E^{\prime}, x_{0}\right)$ and $p \xi_{b_{0}}(y)=b_{0}$ for all $y \in F$. Define a homotopy $h: \sigma^{m} \times I \rightarrow B$ by $h(z, t)=\rho(t)$, and use 2.2 to lift to a homotopy $h_{\rho}:\left(\sigma^{m} \times I, \dot{\sigma}^{m} \times I\right)$ $\rightarrow\left(E, E^{\prime}\right)$ such that $p \tilde{h}_{\rho}=h$ and $\tilde{h}_{\rho} \mid \sigma^{m} \times\{0\}=\xi_{b_{0}} \alpha$. Then $p h_{\rho}(z, 1)=\rho(1)=b_{0}$, so $\tilde{h}_{\rho} \mid \sigma^{m} \times\{1\}:\left(\sigma^{m}, \dot{\sigma}^{m}, z_{0}\right) \rightarrow\left(p^{-1}\left(b_{0}\right), p^{\prime-}{ }_{+}\left(b_{0}\right), x_{1}\right)$, where $x_{1}=\tilde{h}_{\rho}\left(z_{0}, 1\right)$. Thus $\beta=\xi_{b_{0}}^{-1} h_{\rho} \mid \sigma^{m} \times\{1\}$ defines an element of $\pi_{m}\left(F, F^{\prime}, y_{1}\right)$, where $y_{1}=\xi_{b_{0}}^{-1}\left(x_{1}\right)$. The proof that this element depends only on the homotopy classes of $\alpha$ and $\rho$ is the same as usual. Since $\left(F, F^{\prime}\right)$ is $m$-simple, we may disregard base points in $\left(F, F^{\prime}\right)$ and write $\bar{\rho} \circ \bar{\alpha}=\bar{\beta}$, where $\bar{\beta} \in \pi_{m}\left(F, F^{\prime}\right)$ is the homotopy class of $\beta$. In the usual manner, we prove that " $\circ$ " is an operation of $\pi_{1}(B)$ on $\pi_{m}\left(F, F^{\prime}\right)$.

Proposition 2.5. If $\left(F, F^{\prime}\right)$ is m-simple, $\bar{w} \in \pi_{1}\left(E^{\prime}, x_{0}\right), p\left(x_{0}\right)=b_{0}$, $\bar{\alpha} \in \pi_{m}\left(F, F^{\prime}\right)$, and $\bar{\imath}:\left(F, F^{\prime}\right) \rightarrow\left(E, E^{\prime}\right)$ is the inclusion, then

$$
\bar{i}_{*}\left(p_{*}^{\prime}(\bar{w}) \circ \bar{\alpha}\right)=\bar{w} \cdot \bar{i}_{*}(\bar{\alpha}),
$$

where "." is the usual operation of $\pi_{1}\left(E^{\prime}, x_{0}\right)$ on $\pi_{m}\left(E, E^{\prime}, x_{0}\right)$.

Proof. Let $w:\left(\sigma^{1}, \dot{\sigma}^{1}\right) \rightarrow\left(E^{\prime}, x_{0}\right)$ represent $\bar{w}$, and let $\alpha:\left(\sigma^{m}, \dot{\sigma}^{m}, z_{0}\right)$ $\rightarrow\left(F, F^{\prime}, y_{0}\right)$ represent $\bar{\alpha}$. Then $p^{\prime} w$ represents $p_{*}^{\prime}(\bar{w}) \in \pi_{1}\left(B, b_{0}\right)$. As in the discussion above, we construct a homotopy $\tilde{h}_{p^{\prime} w}:\left(\sigma^{m} \times I, \dot{\sigma}^{m} \times I\right) \rightarrow\left(E, E^{\prime}\right)$. 
Let $\mu_{w}(t)=\hbar_{p^{\prime} w}\left(z_{0}, t\right)$. Then $\mu_{w}$ is a curve in $E^{\prime}$ from $x_{0}$ to $x_{1}$. If $\xi_{b_{0}}:\left(F, F^{\prime}\right)$ $\rightarrow\left(E, E^{\prime}\right)$ is an admissible map, and if $\lambda$ is an arbitrary curve in $F^{\prime}$ from $y_{0}$ to $y_{1}=\xi_{b_{a}}^{-1}\left(x_{1}\right)$, then $\lambda^{\prime}=\xi_{b_{0}} \lambda: \sigma^{1} \rightarrow p^{\prime-1}\left(b_{0}\right)$ is a curve from $x_{0}$ to $x_{1}$. Thus $\lambda^{\prime} \bigvee \mu_{w 0}{ }^{-1}=w^{\prime}:\left(\sigma^{1}, \dot{\sigma}^{1}\right) \rightarrow\left(E^{\prime}, x_{0}\right)$ is a closed curve such that $p^{\prime} w^{\prime}=p^{\prime} w$, where " $\bigvee$ " denotes path multiplication. Let $\bar{\beta} \in \pi_{m}\left(F, F^{\prime}, y_{1}\right)$ be the homotopy class of $\xi_{b_{0}}^{-1} h_{p^{\prime} w} \mid \sigma^{m} \times\{1\}$, so that $\bar{i}_{*}(\bar{\beta})=\mu_{w}^{-1 \#} \bar{i}_{*}(\bar{\alpha})$. Thus $i_{*}\left(p_{*}^{\prime}(\bar{w}) \circ \bar{\alpha}\right)=i_{*}\left(\lambda^{\#} \beta\right)$ $=\left(\xi_{b_{0}} \lambda\right)^{\#} i_{*}(\bar{\beta})=\left(\xi_{b_{0}} \lambda\right)^{\#} \mu_{w}^{-1 \#} \bar{i}_{*}(\bar{\alpha})=\bar{w}^{\prime} \cdot \bar{i}_{*}(\bar{\alpha})$. Since $p_{*}^{\prime}(\bar{w})=p_{*}^{\prime}\left(\bar{w}^{\prime}\right), \bar{w}^{-1} \bar{w}^{\prime}=\bar{i}_{*}^{\prime}(\bar{u})$, where $\bar{u} \in \pi_{1}\left(F^{\prime}, y_{0}\right)$. Hence, $\left(\bar{w}^{-1} \bar{w}^{\prime}\right) \cdot \bar{i}_{*}(\bar{\alpha})=i_{*}^{\prime}(\bar{u}) \cdot \bar{i}_{*}(\bar{\alpha})=\bar{i}_{*}(\bar{u} \cdot \bar{\alpha})=\bar{i}_{*}(\bar{\alpha})$, and $\bar{w}^{\prime} \cdot \bar{i}_{*}(\bar{\alpha})=\bar{w} \cdot \bar{i}_{*}(\bar{\alpha})$. Thus $\bar{w} \cdot \bar{i}_{*}(\bar{\alpha})=\bar{w}^{\prime} \cdot \bar{i}_{*}(\bar{\alpha})=\bar{i}_{*}\left(p_{*}^{\prime}(\bar{w}) \circ \bar{\alpha}\right)$.

COROllary 2.6. If $\left(F, F^{\prime}\right)$ is m-simple, then $\pi_{1}(B)$ operates trivially on $\pi_{m}\left(F, F^{\prime}\right)$ if and only if $\left(E, E^{\prime}\right)$ is m-simple.

Proof. Use the formula of 2.5 together with the fact that $\bar{i}_{*}: \pi_{m}\left(F, F^{\prime}\right)$ $\rightarrow \pi_{m}\left(E, E^{\prime}\right)$ is an isomorphism onto.

Proposition 2.7. If $\pi_{i}\left(F, F^{\prime}\right)=0$ for $i<m$ and $\left(F, F^{\prime}\right)$ is $m$-simple, then $i_{*}: H_{m}\left(F, F^{\prime}\right) \rightarrow H_{m}\left(E, E^{\prime}\right)$ is an isomorphism onto if and only if $\pi_{1}(B)$ operates trivially on $\pi_{m}\left(F, F^{\prime}\right)$.

The proof is a combination of the previous corollary and the Hurewicz isomorphism theorem.

3. Obstruction theory. Let $\xi=(E, p, B, F)$ be a fibre space where $B$ is a locally finite simplicial polyhedron. Assume the vertices of $B$ are ordered, and suppose the fibre $F$ is $n$-simple. Let $\pi_{n}(\xi)$ denote the local system of groups on $B$ whose group at $b \in B$ is $\pi_{n}\left(F_{b}\right)$, where $F_{b}=p^{-1}(b)$. For each simplex $\sigma$ of $B$, let $v(\sigma)$ be the first vertex of $\sigma$ and let $F_{\sigma}=F_{v(\sigma)}$. Let $E_{\sigma}=p^{-1}(\sigma)$ as usual. Since $\sigma$ is contractible, $E_{\sigma}$ has the homotopy type of $F_{\sigma}$ and the inclusion map $i_{\sigma}: F_{\sigma} \rightarrow E_{\sigma}$ induces isomorphisms onto $i_{\sigma *}: \pi_{j}\left(F_{\sigma}\right) \rightarrow \pi_{j}\left(E_{\sigma}\right)$.

Now suppose that there is a cross-section $f: B^{n} \cup A \rightarrow E$, where $A$ is a subcomplex of $B$. Let $\sigma$ be an oriented $(n+1)$-simplex of $B-A$. Define $c^{n+1}(f)(\sigma) \in \pi_{n}\left(F_{\sigma}\right)$ to be the element such that $i_{\sigma *}\left(c^{n+1}(f)(\sigma)\right)$ is the element of $\pi_{n}\left(E_{\sigma}\right)$ represented by $f \mid \dot{\sigma}: \dot{\sigma} \rightarrow E$. Thus $c^{n+1}(f)$ is a cochain in $C^{n+1}\left(B, A ; \pi_{n}(\xi)\right)$, which is called the obstruction cochain of $f$. This definition of the obstruction cochain and the following theorem, which gives the main properties of $c^{n+1}(f)$, can all be found in Steenrod's book [16, Part III].

TheOREM 3.1. Let $\xi=(E, p, B, F)$ be a fibre space which admits a crosssection $f: B^{n} \cup A \rightarrow E$, where $A$ is a subcomplex of $B$. Then

(i) $f$ extends to a cross-section $f^{\prime}: B^{n+1} \cup A \rightarrow E$ if and only if $c^{n+1}(f)=0$,

(ii) if $f_{0}, f_{1}: B^{n} \cup A \rightarrow E$ are homotopic cross-sections, then $c^{n+1}\left(f_{0}\right)=c^{n+1}\left(f_{1}\right)$,

(iii) $c^{n+1}(f)$ is a cocycle; we denote its cohomology class by $\bar{c}^{n+1}(f)$,

(iv) $f \mid B^{n-1} \cup A$ extends to a cross-section $f^{\prime}: B^{n+1} \cup A \rightarrow E$ if and only if $\bar{c}^{n+1}(f)=0$,

(v) $\bar{c}^{n+1}(f)$ is a topological invariant. 
Let $\left(\xi, \xi^{\prime}\right)$ be a pair of fibre spaces over the simplicial complex $B$ such that the pair $\left(F, F^{\prime}\right)$ is $(n+1)$-simple, and let $\pi_{n}\left(\xi, \xi^{\prime}\right)$ denote the local system of groups on $B$ whose group at $b \in B$ is $\pi_{n}\left(F_{b}, F_{b}^{\prime}\right)$. Assume the vertices of $B$ are ordered and let $\left(F_{v(\sigma)}, F_{v(\sigma)}^{\prime}\right)=\left(F_{\sigma}, F_{\sigma}^{\prime}\right)$, where $v(\sigma)$ is the first vertex of the simplex $\sigma$.

Suppose there is a cross-section $f: B \rightarrow E$ such that $f \mid B^{n} \cup A: B^{n} \cup A \rightarrow E^{\prime}$, where $A$ is a subcomplex of $B$. We wish to find conditions under which $f$ can be deformed to a cross-section $f^{\prime}: B \rightarrow E$ such that $f^{\prime}\left(B^{n+1} \cup A\right) \subset E^{\prime}$. This leads to a deformation cochain $d^{n+1}(f)$ similar to that of Blakers and Massey [1, p. 187], originally due to Hurewicz (unpublished), but which is adapted to fibre spaces.

Thus let $f:\left(B, B^{n} \cup A\right) \rightarrow\left(E, E^{\prime}\right)$ be a cross-section and let $\sigma$ be an oriented $(n+1)$-simplex of $B-A$. Then $f \mid \sigma:(\sigma, \dot{\sigma}) \rightarrow\left(E_{\sigma}, E_{\sigma}^{\prime}\right)$ and determines an element of $\pi_{n+1}\left(E_{\sigma}, E_{\sigma}^{\prime}\right)$. By Corollary 2.4, the inclusion $i:\left(F_{\sigma}, F_{\sigma}^{\prime}\right) \rightarrow\left(E_{\sigma}, E_{\sigma}^{\prime}\right)$ induces an isomorphism onto $i_{*}: \pi_{n+1}\left(F_{\sigma}, F_{\sigma}^{\prime}\right) \rightarrow \pi_{n+1}\left(E_{\sigma}, E_{\sigma}^{\prime}\right)$. Define $d^{n+1}(f, \sigma)$ $\in \pi_{n+1}\left(F_{\sigma}, F_{\sigma}^{\prime}\right)$ as the element such that $\bar{i}_{*}\left(d^{n+1}(f, \sigma)\right)$ is the homotopy class of $f \mid \sigma$, and define $d^{n+1}(f) \in C^{n+1}\left(B, A ; \pi_{n+1}\left(\xi, \xi^{\prime}\right)\right)$ by $d^{n+1}(f)(\sigma)=d^{n+1}(f, \sigma)$ for each $(n+1)$-simplex $\sigma$ of $B-A$. The cochain $d^{n+1}(f)$ is the deformation cochain of $f$.

Since we will be mainly interested in the connection between $d^{n+1}(f)$ and the obstruction to extending $f^{\prime}=f \mid B^{n} \cup A$ as a cross-section of $\xi^{\prime}$, we omit the proof of the following theorem. The reader is referred to Steenrod's book [16] or Blakers and Massey [1] for the methods of proof.

THEOREM 3.2. Let $\left(\xi, \xi^{\prime}\right)$ be a pair of fibre spaces over the simplicial complex $B$, let $A$ be a subcomplex, and let $f:\left(B, B^{n} \cup A\right) \rightarrow\left(E, E^{\prime}\right)$ be a cross-section. Then

(i) $d^{n+1}(f)=0$ if and only if there is a cross-section $f^{\prime}: B \rightarrow E$ such that $f^{\prime}\left(B^{n+1} \cup A\right) \subset E^{\prime}$ and $f^{\prime}$ is homotopic to $f\left(\right.$ rel $\left.B^{n} \cup A\right)$,

(ii) if $f$ is homotopic to $g$ ( $\mathrm{rel} A$ ), then $d^{n+1}(f)=d^{n+1}(g)$,

(iii) $d^{n+1}(f)$ is a cocycle; its cohomology class will be denoted by $\bar{d}^{n+1}(f)$,

(iv) $\bar{d}^{n+1}(f)$ is a topological invariant,

(v) if $\bar{d}^{n+1}(f)=0$, then $f$ can be deformed (rel $B^{n-1} \cup A$ ) to a cross-section $f^{\prime}: B \rightarrow E$ such that $f^{\prime}\left(B^{n+1} \cup A\right) \subset E^{\prime}$.

The houndary operator $\partial: \pi_{n+1}\left(F, F^{\prime}\right) \rightarrow \pi_{n}\left(F^{\prime}\right)$ in the homotopy sequence of the pair $\left(F, F^{\prime}\right)$ induces a map of local systems $\pi_{n+1}\left(\xi, \xi^{\prime}\right) \rightarrow \pi_{n}(\xi)$, hence a homomorphism

$$
\partial \#: C^{n+1}\left(B, A ; \pi_{n+1}\left(\xi, \xi^{\prime}\right)\right) \rightarrow C^{n+1}\left(B, A ; \pi_{n}\left(\xi^{\prime}\right)\right) .
$$

Proposition 3.3. If $f^{\prime}=f \mid B^{n} \cup A$ is regarded as a cross-section of $\xi^{\prime}$, then

$$
\partial \#\left(d^{n+1}(f)\right)=c^{n+1}\left(f^{\prime}\right) .
$$

Proof. For each $(n+1)$-simplex $\sigma$ of $B$, there is a commutative diagram 


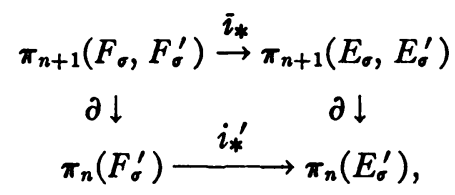

in which the horizontal homomorphisms are isomorphisms onto. If $f \mid \sigma:(\sigma, \dot{\sigma})$ $\rightarrow\left(E_{\sigma}, E_{\sigma}^{\prime}\right)$ represents $\bar{i}_{*}\left(d^{n+1}(f, \sigma)\right)$, then $f \mid \dot{\sigma}: \dot{\sigma} \rightarrow E_{\sigma}^{\prime}$ represents $\partial_{*}\left(d^{n+1}(f, \sigma)\right)$ $=i_{*}^{\prime} \partial\left(d^{n+1}(f, \sigma)\right) \in \pi_{n}\left(E_{\sigma}^{\prime}\right)$. But $i_{*}^{\prime}\left(c^{n+1}\left(f^{\prime}, \sigma\right)\right) \in \pi_{n}\left(E_{\sigma}^{\prime}\right)$ is represented by $f^{\prime}|\dot{\sigma}=f| \dot{\sigma}: \dot{\sigma} \rightarrow E^{\prime}$, so $i_{*}^{\prime}\left(c^{n+1}\left(f^{\prime}, \sigma\right)\right)=i_{*}^{\prime} \partial\left(d^{n+1}(f, \sigma)\right)$, and since $i_{*}^{\prime}$ is an isomorphism, it follows that $\partial\left(d^{n+1}(f, \sigma)\right)=c^{n+1}\left(f^{\prime}, \sigma\right)$, whence $\partial_{\#}\left(d^{n+1}(f)\right)$ $=c^{n+1}\left(f^{\prime}\right)$.

Let $\left(\xi, \xi^{\prime}\right)$ be a pair of fibre spaces such that $\pi_{i}\left(F, F^{\prime}\right)=0$ for $i<n+1$ and such that $\left(F, F^{\prime}\right)$ is $(n+1)$-simple. Also assume that $\pi_{1}(B)$ operates trivially on $\pi_{n+1}\left(F, F^{\prime}\right)$. Under these conditions we will be able to compute the class $\bar{d}^{n+1}(f)$ for a cross-section $f:(B, A) \rightarrow\left(E, E^{\prime}\right)$ and the class $\bar{c}^{n+1}(f \mid A)$, where $f \mid A: A \rightarrow E^{\prime}$ is considered as a cross-section of $\xi^{\prime}$.

Since $\pi_{i}\left(F, F^{\prime}\right)=0$ for $i<n+1$ and $\left(F, F^{\prime}\right)$ is $n$-simple, the Hurewicz homomorphism $\mathfrak{H}: \pi_{i}\left(F, F^{\prime}\right) \rightarrow H_{i}\left(F, F^{\prime}\right)$ is an isomorphism onto for $i \leqq n+1$. By the universal coefficient theorem

$$
H^{n+1}\left(F, F^{\prime} ; \pi_{n+1}\left(F, F^{\prime}\right)\right) \rightarrow \operatorname{Hom}\left(H_{n+1}\left(F, F^{\prime}\right), \pi_{n+1}\left(F, F^{\prime}\right)\right)
$$

is an isomorphism onto. The fundamental class $\theta^{n+1}\left(F, F^{\prime}\right)$ of the pair $\left(F, F^{\prime}\right)$ is the class in $H^{n+1}\left(F, F^{\prime} ; \pi_{n+1}\left(F, F^{\prime}\right)\right)$ corresponding to the inverse of the Hurewicz homomorphism which is in $\operatorname{Hom}\left(H_{n+1}\left(F, F^{\prime}\right), \pi_{n+1}\left(F, F^{\prime}\right)\right)$ under this isomorphism. If $\left(F, F^{\prime}\right)$ is a pair of complexes, $\theta^{n+1}\left(F, F^{\prime}\right)$ turns out to be the first obstruction to contracting $F$ onto $F^{\prime}$.

The fact that $\pi_{1}(B)$ operates trivially on $\pi_{n+1}\left(F, F^{\prime}\right)$ has two important consequences in our computations. First, the coefficient system $\pi_{n+1}\left(\xi, \xi^{\prime}\right)$ is simple, and secondly, the inclusion $i:\left(F, F^{\prime}\right) \rightarrow\left(E, E^{\prime}\right)$ induces isomorphisms onto, $i_{*}: H_{j}\left(F, F^{\prime}\right) \rightarrow H_{j}\left(E, E^{\prime}\right)$ for $j \leqq n+1$, by Proposition 2.7 .

Now suppose that $f:(B, A) \rightarrow\left(E, E^{\prime}\right)$ is a cross-section. Since $\pi_{i}\left(F, F^{\prime}\right)=0$ for $i<n+1, d^{i}(f)=0$ for $i<n+1$, and $f$ can be deformed to a cross-section $\left(B, B^{n} \cup A\right) \rightarrow\left(E, E^{\prime}\right)$, which we again denote by $f$. Let $\sigma$ be an $(n+1)$ simplex of $B-A$, and consider the commutative diagram

$$
\begin{aligned}
& \pi_{n+1}(\sigma, \dot{\sigma}) \stackrel{(f \mid \sigma)_{*}}{\longrightarrow} \pi_{n+1}\left(E, E^{\prime}\right) \stackrel{i_{*}}{\longleftarrow} \pi_{n+1}\left(F, F^{\prime}\right)
\end{aligned}
$$

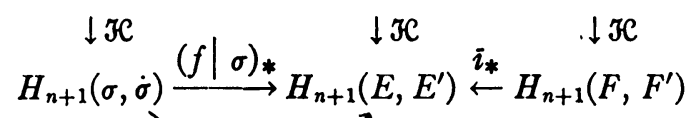

$$
\begin{aligned}
& j^{*}>f^{*} \\
& H_{n+1}\left(B^{n+1}, B^{n}\right)=C_{n+1}(B),
\end{aligned}
$$

where $\mathcal{H}$ is the Hurewicz homomorphism, and $j_{*}$ is induced by the inclusion 
$(\sigma, \dot{\sigma}) \rightarrow\left(B^{n+1}, B^{n}\right)$. Letting $\sigma$ represent the generator of $C_{n+1}(B)$ corresponding to the simplex $\sigma$, i.e., $\sigma$ is the image of a generator of $\pi_{n+1}(\sigma, \dot{\sigma})$ under the composition $j_{*} \mathfrak{H C}$, we see that $i_{*} \mathfrak{H C}\left(d^{n+1}(f, \sigma)\right)=f_{*}(\sigma)$. Let $\pi=\pi_{n+1}\left(F, F^{\prime}\right)$, and consider the commutative diagrams:

$$
\begin{aligned}
& \begin{array}{cc}
H^{n+1}\left(F, F^{\prime} ; \pi\right) & \bar{\imath}^{*} \\
\downarrow & \approx
\end{array} H^{n+1}\left(E, E^{\prime} ; \pi\right) \\
& \operatorname{Hom}\left(H_{n+1}\left(F, F^{\prime}\right), \pi \stackrel{\left(i_{*}, 1\right)}{\approx} \operatorname{Hom}\left(H_{n+1}\left(E, E^{\prime}\right), \pi\right)\right.
\end{aligned}
$$

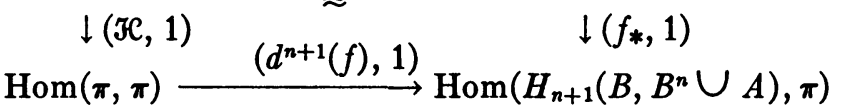

and

$$
\begin{aligned}
\operatorname{Hom}\left(H_{n+1}\left(E, E^{\prime}\right), \pi\right) & \approx H^{n+1}\left(E, E^{\prime} ; \pi\right) \\
\downarrow\left(f_{*}, 1\right) & \approx \quad \downarrow f^{*} \\
\operatorname{Hom}\left(H_{n+1}\left(B, B^{n} \cup A\right), \pi\right) & \approx H^{n+1}\left(B, B^{n} \cup A ; \pi\right)=Z^{n+1}(B, A ; \pi),
\end{aligned}
$$

where 1 denotes the identity map of $\pi$. Using these diagrams and the formula derived above, it is easily checked that

$$
d^{n+1}(f)=f^{*} i^{*-1}\left(\theta^{n+1}\left(F, F^{\prime}\right)\right) .
$$

The commutative diagram

$$
\begin{aligned}
& H^{n+1}\left(F, F^{\prime} ; \pi\right) \stackrel{i^{*}}{\leftrightarrows} H^{n+1}\left(E, E^{\prime} ; \pi\right) \stackrel{k^{*}}{\rightarrow} H^{n+1}\left(E, E_{A}{ }^{\prime} ; \pi\right) \\
& \downarrow f^{*} \\
& H^{n+1}\left(B, B^{n} \cup A ; \pi\right) \stackrel{k^{\prime *}}{\rightarrow} H^{n+1}(B, A ; \pi)
\end{aligned}
$$

together with the fact that $k^{\prime *}: H^{n+1}\left(B, B^{n} \cup A ; \pi\right) \rightarrow H^{n+1}(B, A ; \pi)$ is the natural map of cocycles onto cohomology classes yields the formula

$$
\bar{d}^{n+1}(f)=f^{*} k^{*} i^{*-1}\left(\theta^{n+1}\left(F, F^{\prime}\right)\right) .
$$

Finally, using Proposition 3.3 and the commutative diagram

$$
\begin{array}{cc}
H^{n+1}\left(E, E_{A}{ }^{\prime} ; \pi\right) & \stackrel{\partial_{*}}{\rightarrow} H^{n+1}\left(E, E_{A}^{\prime} ; \pi_{n}\left(\xi^{\prime}\right)\right) \\
\downarrow f^{*} & \downarrow f^{*} \\
H^{n+1}(B, A ; \pi) & \stackrel{\partial_{*}}{\rightarrow} H^{n+1}\left(B, A ; \pi_{n}\left(\xi^{\prime}\right)\right),
\end{array}
$$

where $\partial_{*}$ is the coefficient homomorphism induced by the boundary operator $\partial: \pi_{n+1}\left(F, F^{\prime}\right) \rightarrow \pi_{n}\left(F^{\prime}\right)$, we obtain

$$
\bar{c}^{n+1}(f)=\partial_{*}\left(\bar{d}^{n+1}(f)\right)=f^{*} \partial_{*} k^{*} i^{*-1}\left(\theta^{n+1}\left(F, F^{\prime}\right)\right) .
$$


If $\left(\xi, \xi^{\prime}\right)$ is a pair of fibre spaces satisfying the conditions above and $A$ is a subcomplex of the base $B$, we will denote the class $k^{*} i^{*-1}\left(\theta^{n+1}\left(F, F^{\prime}\right)\right)$ by $\lambda_{A}^{n+1}\left(\xi, \xi^{\prime}\right) \in H^{n+1}\left(E, E_{A}^{\prime} ; \pi\right)$. In case $A$ is empty we write $\lambda^{n+1}\left(\xi, \xi^{\prime}\right)$. Thus we have the formulae

$$
\begin{aligned}
& \bar{d}^{n+1}(f)=f^{*}\left(\lambda_{A}^{n+1}\left(\xi, \xi^{\prime}\right)\right), \\
& \bar{c}^{n+1}(f)=f^{*} \partial_{*}\left(\lambda_{A}^{n+1}\left(\xi, \xi^{\prime}\right)\right) .
\end{aligned}
$$

4. Some properties of principal fibre spaces. The following two theorems give well known properties of principal fibre spaces. The proofs of these theorems may be found in Cartan [6, Exposé 6].

Theorem 4.1. If a principal fibre space $\xi=(E, p, B, G)$ admits a crosssection $f: B \rightarrow E$, then $E$ is homeomorphic to the product space $G \times B$.

Let $\mu: G \times E \rightarrow E$ be the operation of $G$ on $E$ and recall that we write $\mu(g, x)=g \cdot x$.

ThEOREM 4.2. There is a one-to-one correspondence between cross-sections of a principal fibre space $\xi=(E, p, B, G)$ and maps $h: E \rightarrow G$ such that $h(g \cdot x)$ $=g \cdot h(x)$.

The correspondence of 4.2 is defined by assigning to the cross-section $f: B \rightarrow E$ the function $h_{f}: E \rightarrow G$ defined by $h_{f}(x) \cdot f p(x)=x$. It is easily verified that $h_{f}(g \cdot x)=g \cdot h_{f}(x)$.

If $\xi=(E, p, B, G)$ and $\xi^{\prime}=\left(E^{\prime}, p^{\prime}, B^{\prime}, G^{\prime}\right)$ are principal fibre spaces, then a homomorphism of principal fibre spaces $\xi \rightarrow \xi^{\prime}$ is a triple $(\bar{k}, k, \gamma)$ of maps $\bar{k}: E \rightarrow E^{\prime}, k: B \rightarrow B^{\prime}$, and $\gamma: G \rightarrow G^{\prime}$, such that $\gamma$ is a homomorphism of groups, $p^{\prime} \bar{k}=k p$, and $\bar{k}(g \cdot x)=\gamma(g) \cdot \bar{k}(x), x \in E$ and $g \in G$.

Proposition 4.3. If $\xi=(E, p, B, G)$ and $\xi^{\prime}=\left(E^{\prime}, p^{\prime}, B, G^{\prime}\right)$ are principal fibre spaces, if $(\bar{k}, 1, \gamma): \xi \rightarrow \xi^{\prime \prime}$, where 1 is the identity map of $B$, and if $\xi$ admits a cross-section $f: B \rightarrow E$, then $f^{\prime}=\bar{k} f: B \rightarrow E^{\prime}$ is a cross-section, and $\gamma h_{f}=h_{f^{\prime}} \bar{k}$.

Proof. If $b \in B$, then $p^{\prime} f^{\prime}(b)=p^{\prime} \bar{k} f(b)=p f(b)=b$, so $f^{\prime}$ is a cross-section. Let $x \in E$. Then $x=h_{f}(x) \cdot f p(x)$, so $\bar{k}(x)=\bar{k}\left(h_{f}(x) \cdot f p(x)\right)=\gamma h_{f}(x) \cdot \bar{k} f p(x)=\gamma h_{f}(x)$ $\cdot f^{\prime} p(x)$. But $\bar{k}(x)=h_{f}(\bar{k}(x)) \cdot f^{\prime} p^{\prime} \bar{k}(x)=h_{f^{\prime}}(\bar{k}(x)) \cdot f^{\prime} p(x)$, so $h_{f} \cdot \bar{k}(x)=\gamma h_{f}(x)$.

Now suppose $\xi=(E, p, B, G)$ is a principal fibre space, $A \subset B$ is a subcomplex of $B$, and $f_{0}, f_{1}: B \rightarrow E$ are cross-sections such that $f_{0}\left|A=f_{1}\right| A$. Then we have a map $h_{f_{0}}: E \rightarrow G$ satisfying $h_{f_{0}}(x) \cdot f_{0} p(x)=x$, and in particular, if $x=f_{1}(b)$, then $f_{1}(b)=h_{f_{0}}\left(f_{1}(b)\right) \cdot f_{0} p f_{1}(b)=h_{f_{0}}\left(f_{1}(b)\right) \cdot f_{0}(b)$. Define $h_{f_{0} f_{1}}: B \rightarrow G$ as the composition $h_{f_{0} f_{1}}=h_{f_{0}} f_{1}$. Since $f_{0}\left|A=f_{1}\right| A, h_{f_{0} f_{1}}(b)=e$ for $b \in A$, where $e \in G$ is the identity element. Thus

$$
h_{f_{0} f_{1}}:(B, A) \rightarrow(G, e) .
$$


Proposition 4.4. The map $h_{f_{0} f_{1}}: B \rightarrow G$ is homotopic to a constant map (rel $A$ ) if and only if $f_{0}$ and $f_{1}$ are homotopic (rel $A$ ) as cross-sections.

Proof. Let $\Gamma: B \times I \rightarrow G$ be a homotopy such that $\Gamma(b, 0)=h_{f_{0} f_{1}}(b), \Gamma(b, t)$ $=e \in G$ for $(b, t) \in A \times I \cup B \times\{1\}$. Define $H: B \times I \rightarrow E$ by the formula $H(b, t)=\Gamma(b, t) \cdot f_{0}(b)$. It is easily checked that $H(b, t)=f_{0}(b)$ for $(b, t) \in A \times I$ $\cup B \times\{1\}, H(b, 0)=f_{1}(b)$, and $p H(b, t)=b$.

Suppose that $f_{t}: B \rightarrow E, 0 \leqq t \leqq 1$, is a homotopy of $f_{0}$ to $f_{1}$ such that $f_{t}\left|A=f_{0}\right| A, 0 \leqq t \leqq 1$. Define $\Gamma: B \times I \rightarrow G$ by $\Gamma(b, t)=h_{f_{0} f_{1-t}}(b)$. Then $\Gamma(b, 0)$ $=h_{f_{0} f_{1}}(b), \Gamma(b, 1)=e$, and $\Gamma(A \times I)=e$,

This proposition shows that the homotopy classification of cross-sections of a principal fibre space $\xi=(E, p, B, G)$ which agree on a subcomplex $A \subset B$ can be stated in terms of deforming maps $(B, A) \rightarrow(G, e)$ to maps $B \rightarrow\{e\}$ $(\operatorname{rel} A)$.

Let $\xi=(E, p, B, G)$ be a principal fibre space, let $A \subset B$, and let $f_{0}, f_{1}: B^{n}$ $\cup A \rightarrow E$ be cross-sections such that $f_{0}\left|B^{n-1} \cup A=f_{1}\right| B^{n-1} \cup A$. A function $h_{f_{0}}: X^{n}=p^{-1}\left(B^{n} \cup A\right) \rightarrow G$ is defined, so the function $h_{f_{0} f_{1}}:\left(B^{n} \cup A, B^{n-1} \cup A\right)$ $\rightarrow(G, e)$ is defined. If $\sigma$ is an oriented $n$-simplex of $B-A$, let $d^{n}\left(f_{0}, f_{1}, \sigma\right)$ $\in \pi_{n}(G, e)$ be the homotopy class of the map $h_{f_{0} f_{1}} \mid \sigma:(\sigma, \dot{\sigma}) \rightarrow(G, e)$, and define an $n$-cochain $d^{n}\left(f_{0}, f_{1}\right) \in C^{n}\left(B, A ; \pi_{n}(G, e)\right)$ by $d^{n}\left(f_{0}, f_{1}\right)(\sigma)=d^{n}\left(f_{0}, f_{1}, \sigma\right)$. It is easily seen that this cochain is the same as the one defined by Steenrod [16, p. 169]. Proofs of the following properties of $d^{n}\left(f_{0}, f_{1}\right)$ can be found in Steenrod [16, pp. 169-177], however the proofs are somewhat simpler in the case of a principal bundle if we use the definition given above.

Theorem 4.5. If $\xi=(E, p, B, G)$ is a principal fibre space which admits cross-sections $f_{0}, f_{1}: B^{n} \cup A \rightarrow E$ such that $f_{0}\left|B^{n-1} \cup A=f_{1}\right| B^{n-1} \cup A$, then

(i) $d^{n}\left(f_{0}, f_{1}\right)=0$ if and only if $f_{0}$ is homotopic to $f_{1}\left(\operatorname{rel} B^{n-1} \cup A\right)$,

(ii) $\delta d^{n}\left(f_{0}, f_{1}\right)=c^{n+1}\left(f_{0}\right)-c^{n+1}\left(f_{1}\right)$,

(iii) if $f_{2}: B^{n} \cup A \rightarrow E$ is a cross-section such that $f_{2}\left|B^{n-1} \cup A=f_{0}\right| B^{n-1} \cup A$, then $d^{n}\left(f_{0}, f_{1}\right)+d^{n}\left(f_{1}, f_{2}\right)+d^{n}\left(f_{2}, f_{0}\right)=0$,

(iv) if $f_{0}: B^{n} \cup A \rightarrow E$ and $d \in C^{n}\left(B, A ; \pi_{n}(G)\right)$, then $f_{0} \mid B^{n-1} \cup A$ can be extended to $f_{1}: B^{n} \cup A \rightarrow E$ with $d^{n}\left(f_{0}, f_{1}\right)=d$,

(v) if $f_{0}$ and $f_{1}$ extend to $B^{n+1} \cup A$ so that $d^{n}\left(f_{0}, f_{1}\right)$ is a cocycle, then the cohomology class $\bar{d}^{n}\left(f_{0}, f_{1}\right)$ is a topological invariant and its vanishing is necessary and sufficient for $f_{0}$ and $f_{1}$ to be homotopic (rel $B^{n-2} \cup A$ ).

Proposition 4.6. If $\xi=(E, p, B, G)$ is a principal fibre space for which $\pi_{i}(G)=0,0 \leqq i<n$, if $f_{0}, f_{1}: B^{n+1} \rightarrow E$ are cross-sections such that $f_{0} \mid B^{n-1}$ $=f_{1} \mid B^{n-1}$, so that $h_{f_{0} f_{1}}:\left(B^{n+1}, B^{n-1}\right) \rightarrow(G, e)$ is defined, and if $\theta^{n}(G) \in H^{n}\left(G ; \pi_{n}(G)\right)$ is the fundamental class of $G$, then

$$
h_{f_{0} f_{1}}^{*}\left(\theta^{n}(G)\right)=\bar{d}^{n}\left(f_{0}, f_{1}\right) \in H^{n}\left(B ; \pi_{n}(G)\right) .
$$

The proof will be omitted, since it is analogous to the discussion at the end of $\$ 3$. 
For the sequence of propositions which follows, we need some lemmas on the cohomology of product spaces. Let $X$ and $Y$ be spaces, $x_{0} \in X, y_{0} \in Y$, and let $X \bigvee Y=X \times\left\{y_{0}\right\} \cup\left\{x_{0}\right\} \times Y$, with inclusion $j: X \vee Y \rightarrow X \times Y$. Let the maps $i_{1}, i_{2}, p_{1}, p_{2}$, be defined as follows: $i_{1}: X \rightarrow X \vee Y, i_{1}(x)=\left(x, y_{0}\right) ; i_{2}: Y$ $\rightarrow X \bigvee Y, i_{2}(y)=\left(x_{0}, y\right) ; p_{1}: X \times Y \rightarrow X, p_{1}(x, y)=x ;$ and $p_{2}: X \times Y \rightarrow Y$, $p_{2}(x, y)=y$. Clearly $p_{1} j i_{1}=$ identity on $X$ and $p_{2} j i_{2}=$ identity on $Y$. This notation will hold throughout this section and in $\$ 6$.

LEMMA 4.7. If $\pi$ is an abelian group, then for each $i>0$,

$$
H^{i}(X \vee Y ; \pi) \approx H^{i}(X ; \pi)+H^{i}(Y ; \pi) \text { (direct sum). }
$$

The proof is very easy.

Lемма 4.8. For each $i>0$, the following sequence is exact and splits.

$$
0 \rightarrow H^{i}(X \times Y, X \vee Y ; \pi) \stackrel{k^{*}}{\rightarrow} H^{i}(X \times Y ; \pi) \stackrel{j^{*}}{\rightarrow} H^{i}(X \vee Y ; \pi) \rightarrow 0
$$

Proof. Consider the cohomology sequence of the pair $(X \times Y, X \vee Y)$ and the diagram

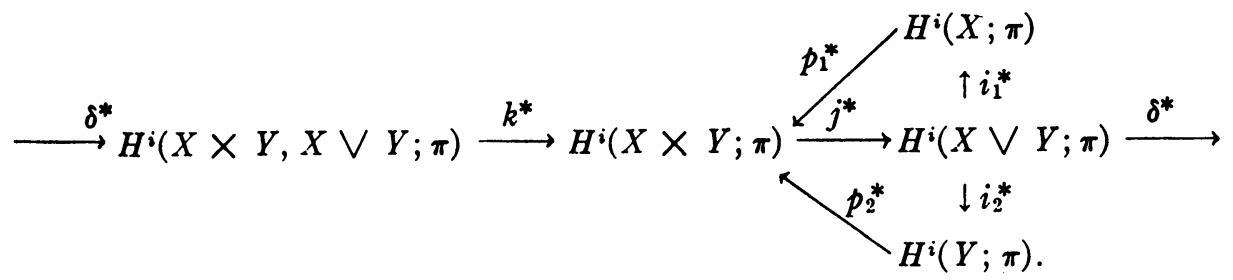

The splitting homomorphism is $\theta(w)=p_{1}^{*} i_{1}^{*}(w)+p_{2}^{*} i_{2}^{*}(w), w \in H^{i}(X \bigvee Y ; \pi)$, i.e., there is a homomorphism

$$
\chi: H^{i}(X \times Y ; \pi) \rightarrow H^{i}(X \times Y, X \vee Y ; \pi)
$$

such that each element $u \in H^{i}(X \times Y ; \pi)$ can be written uniquely in the form

$$
u=p_{1}^{*} i_{1}^{*} j^{*}(u)+p_{2}^{*} i_{2}^{*} j^{*}(u)+k^{*} \chi(u) \text {. }
$$

Lemma 4.9. If $f_{1}:\left(X^{\prime}, x_{0}^{\prime}\right) \rightarrow\left(X, x_{0}\right)$ and $f_{2}:\left(Y^{\prime}, y_{0}^{\prime}\right) \rightarrow\left(Y, y_{0}\right)$, then the following diagram is commutative:

$$
\begin{aligned}
& 0 \rightarrow B^{i}(X \times Y, X \vee Y ; \pi) \stackrel{k^{*}}{\rightarrow} H^{i}(X \times Y ; \pi) \underset{\theta}{\stackrel{j^{*}}{\rightleftarrows}} H^{i}(X \vee Y ; \pi) \rightarrow 0 \\
& \downarrow\left(f_{1} \times f_{2}\right)^{*} \quad \downarrow\left(f_{1} \times f_{2}\right)^{*} \quad \downarrow\left(f_{1} \vee f_{2}\right)^{*} \\
& 0 \rightarrow H^{i}\left(X^{\prime} \times Y^{\prime}, X^{\prime} \vee Y^{\prime} ; \pi \stackrel{k^{\prime *}}{\rightarrow} H^{i}\left(X^{\prime} \times Y^{\prime} ; \pi\right) \underset{\theta^{\prime}}{\stackrel{j^{*}}{\rightleftarrows}} H^{i}\left(X^{\prime} \vee Y^{\prime} ; \pi\right) \rightarrow 0\right. \text {, }
\end{aligned}
$$

i.e., the splitting of 4.8 is natural.

The proof is easy. 
Let $\xi=(E, p, B, G)$ be a principal fibre space with $\mu: G \times E \rightarrow E$ the operation of $G$ on $E$. Let $b_{0}$ be a vertex of $B, x_{0} \in p^{-1}\left(b_{0}\right)$, and let $e \in G$ be the identity. By $G \bigvee E$, we mean $G \times\left\{x_{0}\right\} \cup\{e\} \times E$, and $i: G \rightarrow E$ will denote the inclusion of the fibre $i(g)=g \cdot x_{0} \in p^{-1}\left(b_{0}\right)$ for $g \in G$. Consider the diagram

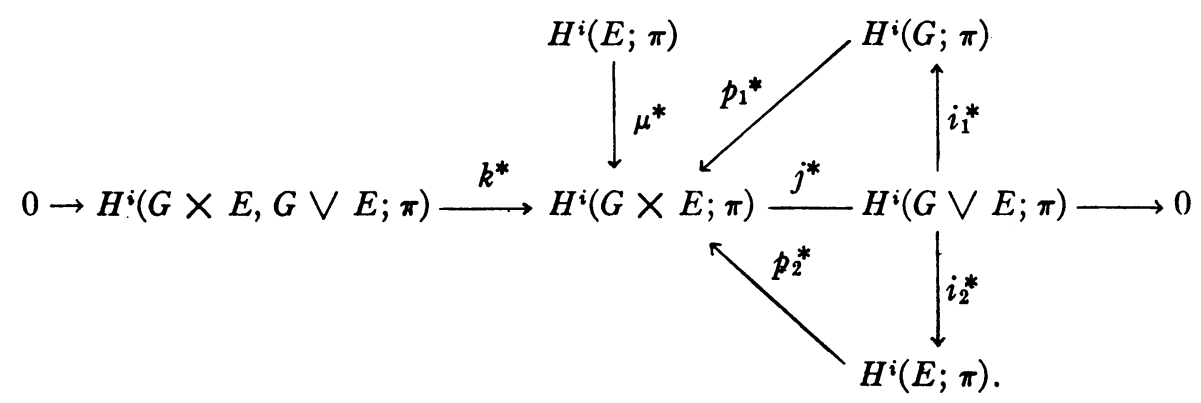

Proposition 4.10. There exists a homomorphism

$$
\chi^{\prime}: H^{i}(E ; \pi) \rightarrow H^{i}(G \times E, G \vee E ; \pi)
$$

such that if $u \in H^{i}(E ; \pi)$, then

$$
\mu^{*}(u)=p_{1}^{*} i^{*}(u)+p_{2}^{*}(u)+k^{*} \chi^{\prime}(u) .
$$

Proof. $\mu j i_{1}(g)=\mu\left(g, x_{0}\right)=i(g)$ and $\mu j i_{2}(x)=\mu(e, x)=x$, so $p_{1}^{*} i_{1}^{*} j^{*} \mu^{*}(u)$ $=p_{1}^{*} i^{*}(u)$ and $p_{2}^{*} i_{2}^{*} j^{*} \mu^{*}(u)=p_{2}^{*}(u)$. From Lemma 4.8 we obtain $\mu^{*}(u)$ $=p_{1}^{*} i^{*}(u)+p_{2}^{*}(u)+k^{*} \chi \mu^{*}(u)=p_{1}^{*} i^{*}(u)+p_{2}^{*}(u)+k^{*} \chi^{\prime}(u)$, where $\chi^{\prime}$ is the composition $\chi^{\prime}=\chi \mu^{*}$.

5. The decomposition of a principal fibre bundle. We now restrict our attention to principal fibre bundles $\xi=(E, p, B, G, G)$ for which the group $G$ is a connected countable CW-group. The following theorem is due to Milnor [14].

THEOREM 5.1. If $G$ is a countable $C W$-group, then there is a universal principal fibre bundle $\xi(G)=\left(E_{G}, \pi, B_{G}, G, G\right)$ which has the properties

(i) $E_{G}$ is a contractible space,

(ii) $B_{G}$ is a countable $C W$-complex.

Recall that any principal $G$-bundle over a base space $B$ is induced by a continuous map $B \rightarrow B_{G}$, and homotopic maps induce equivalent bundles.

Proposition 5.2. If $G$ is a connected countable $C W$-group, then for any integer $N \geqq 1$, there is a sequence of $C W$-groups and continuous homomorphisms

$$
\tilde{G}=G_{N+1} \stackrel{\gamma_{N}}{\longrightarrow} G_{N} \stackrel{\gamma_{N-1}}{\longrightarrow} G_{N-1} \rightarrow \cdots \rightarrow G_{2} \stackrel{\gamma_{1}}{\longrightarrow} G_{1},
$$

such that

(i) $\gamma_{n}: G_{n+1} \rightarrow G_{n}$ embeds $G_{n+1}$ as a subcomplex of $G_{n}$ for $n=1,2, \cdots, N$, hence is a continuous isomorphism into, 
(ii) there is a continuous homomorphism $\gamma^{\prime}: \widetilde{G}=G_{\widehat{N}+1} \rightarrow G$ which is part of a homotopy equivalence of $\widetilde{G}$ and $G$,

(iii) $\pi_{i}\left(G_{n}\right)=0$ for $i>n$ and $n=1,2, \cdots, N$,

(iv) if $\eta_{n}: G \rightarrow G_{n}$ is the composition $\eta_{n}=\gamma_{n} \gamma_{n+1} \cdots \gamma_{N}$, then $\eta_{n^{*}}: \pi_{i}(G)$ $\rightarrow \pi_{i}\left(G_{n}\right)$ is an isomorphism onto for $i \leqq n$ and $n=1,2, \cdots, N$.

Thus this sequence of groups and homomorphisms is a (finite) Postnikov system for the group $G$.

Proof. Since $B_{G}$ is a countable $\mathrm{CW}$-complex, it has the same homotopy type as a simplicial complex [17, Theorem 13], and we can induce a universal bundle over this simplicial complex. Thus we may as well assume that $B_{G}$ itself is a countable simplicial complex. Let $X_{N+1}=B_{G}$, and let $X_{N}$ be the space obtained from $B_{G}$ by attaching cells of dimension $\geqq N+3$ so as to "kill" $\pi_{N+2}\left(B_{G}\right), \pi_{N+3}\left(B_{G}\right), \cdots$. By using the simplicial approximation theorem, we may assume that the cells are attached to $B_{G}$ by simplicial maps. By $\left[17\right.$, Lemma 2, p. 239], there is a simplicial subdivision of $X_{N}$ which contains $B_{G}=X_{N+1}$ as a subcomplex, i.e., we may regard the inclusion $j_{N}: B_{G}$ $=X_{N+1} \rightarrow X_{N}$ as a simplicial map. We now repeat this process on $X_{N}$ to obtain $X_{N-1}$, and so on, until we obtain a sequence of spaces and maps

$$
B_{G}=X_{N+1} \stackrel{j_{N}}{\longrightarrow} X_{N} \stackrel{j_{N-1}}{\longrightarrow} X_{N-1} \rightarrow \cdots \rightarrow X_{2} \stackrel{j_{1}}{\longrightarrow} X_{1},
$$

where each $X_{n}$ is a simplicial complex, and the maps $j_{n}: X_{n+1} \rightarrow X_{n}, n=1,2$, $\cdots, N$, are simplicial inclusion maps.

The maps $k_{n}=j_{n} j_{n+1} \cdots j_{N}: X_{N+1} \rightarrow X_{N}$ induce isomorphisms onto $k_{n^{*}}: \pi_{i}\left(X_{N+1}\right) \rightarrow \pi_{i}\left(X_{n}\right)$ for $i \leqq n+1$, and $\pi_{i}\left(X_{n}\right)=0$ for $i>n+1$ and $n=1,2, \cdots, N$ by the construction. Following Milnor [13], for each $n, n$ $=1,2, \cdots, \mathrm{N}+1$, we constructa principal bundle $\xi\left(G_{n}\right)=\left(\tilde{X}_{n}, \pi_{n}, X_{n}, G_{n}, G_{n}\right)$, where $G_{n}$ is a countable CW-group and $\tilde{X}_{n}$ is a contractible CW-complex. Recall that in Milnor's construction $\tilde{X}_{n}$ is a space of equivalence classes of polygonal paths in $X_{n}$ which begin at a fixed vertex of $X_{n}$, and that $G_{n}$ is the set of those equivalence classes of such paths which begin and end at the same vertex. Choose a fixed vertex of $B_{G}=X_{N+1}$ as the fixed vertex in this construction. Then the simplicial inclusion maps $X_{n+1} \rightarrow X_{n}$ induce one-to-one bundle maps $\tilde{X}_{n+1} \rightarrow \tilde{X}_{n}$, and it is easily checked that the induced maps $G_{n+1} \rightarrow G_{n}$ are continuous isomorphisms into. Following through Milnor's proof that $G_{n}$ is a CW-complex and using the fact that $j_{n}: X_{n+1} \rightarrow X_{n}$ is a simplicial inclusion map, we find that $G_{n+1}$ is a subcomplex of $G_{n}$. Setting $G_{N+1}=\tilde{G}$, we have proved (i). By another theorem of Milnor [13, p. 282], the bundle $\xi(G)=\left(E_{G}, \pi, B_{G}, G, G\right)$ is induced by a continuous homomorphism $\gamma^{\prime}: \widetilde{G} \rightarrow G$, and $\gamma^{\prime}$ is part of a homotopy equivalence of $\widetilde{G}$ and $G$, so (ii) is proved. Since each $\tilde{X}_{n}$ is contractible, it follows that $\pi_{i+1}\left(X_{n}\right) \approx \pi_{i}\left(G_{n}\right)$, so $\pi_{i}\left(G_{n}\right)=0$ for $i>n$, and (iii) is proved. To prove (iv), consider the bundle homomorphism $\left(\bar{k}_{n}, k_{n}, \gamma_{n}\right): \xi\left(G_{N+1}\right) \rightarrow \xi\left(G_{n}\right)$. We have a commutative diagram 


$$
\begin{gathered}
\pi_{i+1}\left(X_{N+1}\right) \stackrel{k_{n^{*}}}{\longrightarrow} \pi_{i+1}\left(X_{n}\right) \\
\downarrow \approx \\
\pi_{i}\left(G_{N+1}\right) \stackrel{\eta_{n}^{*}}{\longrightarrow} \pi_{i}\left(G_{n}\right)
\end{gathered}
$$

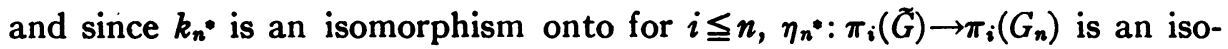
morphism onto for $i \leqq n$.

REMARK. The homomorphism $\gamma^{\prime}: \widetilde{G} \rightarrow G$ gives rise to a bundle homomorphism $\left(g^{\prime}, 1, \gamma^{\prime}\right)$ of $\xi(\widetilde{G})=\left(\tilde{X}_{N+1}, \pi^{\prime}, B_{G}, \widetilde{G}, \widetilde{G}\right)$ to $\xi(G)=\left(E_{G}, \pi, B_{G}, G, G\right)$, where 1 denotes the identity map of $B_{a}$. Let $\xi=(E, p, B, G, G)$ be a principal $G$ bundle, and let $\phi_{\xi}: B \rightarrow B_{G}$ be a classifying map. Define $\phi_{\xi}^{-1}(\xi(\widetilde{G}))=\xi^{\prime}$ $=\left(E^{\prime}, p^{\prime}, B, G, G\right)$. Then there is a homomorphism $\left(h, 1, \gamma^{\prime}\right): \xi^{\prime} \rightarrow \xi$ induced by $\left(g^{\prime}, 1, \gamma^{\prime}\right)$. Thus there is a commutative diagram

$$
\begin{gathered}
\rightarrow \pi_{i+1}(B) \rightarrow \pi_{i}(\tilde{G}) \rightarrow \pi_{i}\left(E^{\prime}\right) \rightarrow \pi_{i}(B) \rightarrow \pi_{i-1}(\tilde{G}) \rightarrow \\
\quad \downarrow \approx \gamma_{*}^{\prime} \quad \downarrow h_{*}^{\prime} \quad \downarrow \approx \quad \downarrow \gamma_{*}^{\prime} \\
\rightarrow \pi_{i+1}(B) \rightarrow \pi_{i}(G) \rightarrow \pi_{i}(E) \rightarrow \pi_{i}(B) \rightarrow \pi_{i-1}(G) \rightarrow,
\end{gathered}
$$

in which the rows are exact, and since $\boldsymbol{\gamma}^{\prime}$ is part of a homotopy equivalence, an application of the "fives lemma" yields the fact that the induced map $h_{*}^{\prime}: \pi_{i}\left(E^{\prime}\right) \rightarrow \pi_{i}(E)$ is an isomorphism onto for all $i$. It follows that the maps $h_{*}^{\prime}: H_{*}\left(E^{\prime}\right) \rightarrow H_{*}(E)$ and $h^{\prime *}: H^{*}\left(E^{\prime}\right) \rightarrow H^{*}(E)$ are isomorphisms onto. Moreover, if either $\xi^{\prime}$ or $\xi$ admits a cross-section over the $n$-skeleton $B^{n}$ of $B$, then $f_{\xi} \mid B^{n}: B^{n} \rightarrow B_{G}$ is homotopic to a constant map. This shows that the crosssection problems in $\xi^{\prime}$ and $\xi$ are equivalent. From now on, we will assume without explicitly mentioning it that we have replaced the bundle $\xi$ by the bundle $\xi^{\prime}$, i.e., if $\xi=(E, p, B, G, G)$ is a principal bundle, we assume that $G$ has a Postnikov system $G \rightarrow G_{N} \rightarrow \cdots \rightarrow G_{1}$ in which each map is a continuous isomorphism into.

TheOREM 5.3. Let $G$ be a countable connected $C W$-group, and let $\xi=(E, p, B, G, G)$ be a principal bundle over the $C W$-complex $B$. Then for each integer $N \geqq 1$, there is a sequence of principal bundles $\xi_{n}=\left(E_{n}, p_{n}, B, G_{n}, G_{n}\right)$, $n=1,2, \cdots, N$, a sequence of bundle homomorphisms $\left(g_{n}, 1, \gamma_{n}\right): \xi_{n+1} \rightarrow \xi_{n}$, $n=1,2, \cdots, N-1$, and a bundle homomorphism $\left(g_{N}, 1, \gamma_{N}\right): \xi \rightarrow \xi_{N}$ such that

(i) each $\left(g_{n}, 1, \gamma_{n}\right)$ embeds $\xi_{n+1}$ in $\xi_{n}$ as a fibre subspace and $\left(g_{N}, 1, \gamma_{N}\right)$ embeds $\xi$ in $\xi_{N}$ as a fibre subspace,

(ii) if $h_{n}=g_{n} g_{n+1} \cdots g_{N}: E \rightarrow E_{n}$, then $h_{n}{ }^{*}: \pi_{i}(E) \rightarrow \pi_{i}\left(E_{n}\right)$ is an isomorphism for $i \leqq n$ and is onto for $i \leqq n+1$,

(iii) $\pi_{i}\left(E_{n-1}, E_{n}\right)=0$ if $i \neq n+1$ and $\pi_{n+1}\left(E_{n-1}, E_{n}\right)=\pi_{n}(G)$.

Proof. Let $\left\{g_{i j}\right\}$ be the set of coordinate transformations of the bundle $\xi$. Let $\xi_{N}$ be the $G_{N}$ bundle over $B$ constructed by using the collection $\left\{\gamma_{N} g_{i j}\right\}$ as coordinate transformations and Theorem 3.2 of Steenrod [16]. If $\xi_{n+1}$ has 
been constructed and has coordinate transformations $\left\{g_{i j}\right\}$, we construct $\xi_{n}$ using as coordinate transformations the collection $\left\{\gamma_{n} g_{i j}\right\}$. It is easily seen from the construction that the induced bundle homomorphisms $\left(g_{n}, 1, \gamma_{n}\right)$, $n=1,2, \cdots, N$, are one-to-one, and $\xi_{n}$ is a fibre subspace of $\xi_{n-1}$ and $\xi$ is a fibre subspace of $\xi_{N}$. Thus the existence of the bundles and the homomorphisms is proved as well as (i). To prove (ii), consider the homotopy ladder

$$
\begin{aligned}
& \rightarrow \pi_{i+1}(B) \rightarrow \pi_{i}(G) \rightarrow \pi_{i}(E) \rightarrow \pi_{i}(B) \rightarrow \pi_{i-1}(G) \rightarrow \\
& \downarrow \approx \quad \downarrow \eta_{n^{*}} \quad \downarrow h_{n^{*}} \quad \downarrow \approx \downarrow \eta_{n^{*}} \\
& \rightarrow \pi_{i+1}(B) \rightarrow \pi_{i}\left(G_{n}\right) \rightarrow \pi_{i}\left(E_{n}\right) \rightarrow \pi_{i}(B) \rightarrow \pi_{i-1}\left(G_{n}\right) \rightarrow
\end{aligned}
$$

induced by the bundle homomorphism $\left(h_{n}, 1, \eta_{n}\right): \xi \rightarrow \xi_{n}$. Since $\eta_{n}^{*}: \pi_{i}(G)$ $\rightarrow \pi_{i}\left(G_{n}\right)$ is an isomorphism onto for $i \leqq n$ and $\pi_{i}\left(G_{n}\right)=0$ for $i>n$, the "fives lemma" yields the result of (ii). Part (iii) follows when we observe that from the homotopy sequence of the pair $\left(G_{n-1}, G_{n}\right), \pi_{n+1}\left(G_{n-1}, G_{n}\right) \approx \pi_{n}\left(G_{n}\right) \approx \pi_{n}(G)$, and $\pi_{i}\left(G_{n-1}, G_{n}\right)=0$ otherwise. Applying Lemma 2.4 , the inclusion $i:\left(G_{n-1}, G_{n}\right)$ $\rightarrow\left(E_{n-1}, E_{n}\right)$ induces an isomorphism onto $i_{*}: \pi_{j}\left(G_{n-1}, G_{n}\right) \rightarrow \pi_{j}\left(E_{n-1}, E_{n}\right)$ for all $j$.

A quadruple $\left(\xi, N,\left\{\xi_{n}\right\},\left\{\left(g_{n}, 1, \gamma_{n}\right)\right\}\right)$ satisfying the conditions of this theorem will be called a decomposition of $\xi$ of length $N$.

Lemma 5.4. Let $\xi=(E, p, B, G, G)$ be a principal G-bundle and let $\left(\xi, N,\left\{\xi_{n}\right\},\left\{\left(g_{n}, 1, \gamma_{n}\right)\right\}\right)$ be a decomposition of $\xi$. Suppose $f: B^{\prime} \rightarrow B$, and let $f^{-1}(\xi)$ be the induced bundle. Then the induced bundles $f^{-1}\left(\xi_{n}\right), n=1,2, \cdots, N$, are the bundles in a decomposition of $f^{-1}(\xi)$.

Proof. Let $\left\{V_{i}\right\}$ be the coordinate neighborhoods of $\xi$ and let $\left\{g_{i j}\right\}$ be the coordinate transformations of $\xi$. Then those of $f^{-1}(\xi)$ are $\left\{f^{-1}\left(V_{i}\right)\right\}$ and $\left\{g_{i j} f\right\}$ respectively. If $\eta_{n}: G \rightarrow G_{n}$ is the homomorphism defined in 5.2, $\left(f^{-1}(\xi)\right)_{n}$. has coordinate transformations $\left\{\eta_{n} g_{i j} f\right\}$. But $\xi_{n}$ has coordinate transformations $\left\{\eta_{n} g_{i j}\right\}$, so those of $f^{-1}\left(\xi_{n}\right)$ are $\left\{\eta_{n} g_{i j} f\right\}$. Thus $\left(f^{-1}(\xi)\right)_{n}$ $=f^{-1}\left(\xi_{n}\right)$.

In particular, if we choose a fixed decomposition

$$
\left(\xi(G), N,\left\{\tilde{\xi}_{n}\right\},\left\{\left(\tilde{g}_{n}, 1, \gamma_{n}\right)\right\}\right)
$$

for the universal $G$-bundle $\xi(G)$, then for any $G$-bundle $\xi$, a decomposition $\left(\xi, N,\left\{\xi_{n}\right\},\left\{\left(g_{n}, 1, \gamma_{n}\right)\right\}\right)$ is induced by a classifying map $\phi_{\xi}: B \rightarrow B_{G}$. It is also clear that the bundle $\xi_{n}=\left(\tilde{E}_{n}, \tilde{p}_{n}, B_{G}, G_{n}, G_{n}\right)$ in a decomposition of $\xi(G)$ is induced by the map $k_{n}: B_{G} \rightarrow X_{n}$ of 5.2.

Proposition 5.5. Let $\xi=(E, p, B, G, G)$ be a principal bundle over a $C W$ complex $B$. Then there exists a cross-section $f: B^{n} \rightarrow E$ if and only if there is a cross-section $f^{\prime}: B \rightarrow E_{n-1}$, where $\xi_{n-1}=\left(E_{n-1}, p_{n-1}, B, G_{n-1}, G_{n-1}\right)$ is a term in a decomposition of $\xi$ of length $N \geqq n-1$. 
Proof. Suppose $f: B^{n} \rightarrow E$ is a cross-section. Then $h_{n-1}: f B^{n} \rightarrow E_{n-1}$ is a crosssection because $p_{n-1} h_{n-1} f(b)=p f(b)=b$ for $b \in B^{n}$. The obstructions to extending $h_{n-1} f$ will be in the groups $H^{k}\left(B ; \pi_{k-1}\left(G_{n-1}\right)\right)$ for $k \geqq n+1$. But $\pi_{k-1}\left(G_{n-1}\right)=0$ for $k \geqq n+1$, so all higher obstructions to the extension of $h_{n-1} f$ vanish, and $h_{n-1} f$ extends to a cross-section $f^{\prime}: B \rightarrow E_{n-1}$. Observe that $h_{n-1} f=f^{\prime} \mid B^{n}$.

Now suppose that $f^{\prime}: B \rightarrow E_{n-1}$ is a cross-section. Let $\phi_{\xi}: B \rightarrow B_{G}$ be a classifying map for $\xi$. Then $\xi_{n-1}=\phi_{\xi}^{-1}\left(\xi_{n-1}\right)=\left(k_{n-1} \phi_{\xi}\right)^{-1}\left(\xi\left(G_{n-1}\right)\right)$, where $\xi\left(G_{n-1}\right)=\left(\tilde{X}_{n-1}, \pi, X_{n-1}, G_{n-1}, G_{n-1}\right)$ as in 5.2, i.e., $k_{n-1} \phi_{\xi}: B \rightarrow X_{n-1}$ is a classifying map for $\xi_{n-1}$. But $\xi_{n-1}$ admits a cross-section, so $k_{n-1} \phi_{\xi}$ is homotopic to a constant map. Since $k_{n-1}: \pi_{i}\left(B_{G}\right) \rightarrow \pi_{i}\left(X_{n-1}\right)$ is an isomorphism onto for $i \leqq n$, it follows that $\phi_{\xi^{*}:} \pi_{i}(B) \rightarrow \pi_{i}\left(B_{G}\right)$ is the zero homomorphism for $i \leqq n$. Thus $\phi_{\xi} \mid B^{n}: B^{n} \rightarrow B_{G}$ is homotopic to a constant map and $\xi_{B^{n}}$ is a trivial bundle. Thus there is a cross section $f: B^{n} \rightarrow E$.

We remark that the decomposition of a principal bundle will enter our computation of obstructions in a manner similar to the way in which the Moore-Postnikov system enters the computation of obstructions as done by Hermann [9].

Let $\left(\xi, N,\left\{\xi_{n}\right\},\left\{\left(g_{n}, 1, \gamma_{n}\right)\right\}\right)$ be a decomposition of $(E, p, B, G, G)=\xi$, where $B$ is a locally finite polyhedron. Then each $\left(\xi_{n-1}, \xi\right)$ is a pair of fibre spaces, and $\pi_{i}\left(G_{n-1}, G\right)=0$ for $i<n+1$. From the fact that $\left(G_{n-1}, G\right)$ is a pair of connected group complexes and $\partial: \pi_{n+1}\left(G_{n-1}, G\right) \rightarrow \pi_{n}(G)$ is an isomorphism onto, it follows that $\left(G_{n-1}, G\right)$ is $(n+1)$-simple and $\pi_{1}(B)$ operates trivially on $\pi_{n+1}\left(G_{n-1}, G\right)$. Under these conditions, if $A$ is a subcomplex of $B$, the class $\lambda_{A}^{n+1}\left(\xi_{n-1}, \xi\right) \in H^{n+1}\left(E_{n-1}, E_{A} ; \pi_{n+1}\left(G_{n-1}, G\right)\right)$ is defined as in $\S 3$. We define a new class $\lambda_{A}^{n+1}(\xi) \in H^{n+1}\left(E_{n-1}, E_{A} ; \pi_{n}(G)\right)$ as the image of $\lambda_{A}^{n+1}\left(\xi_{n-1}, \xi\right)$ under the isomorphism

$$
\partial_{*}: H^{n+1}\left(E_{n-1}, E_{A} ; \pi_{n+1}\left(G_{n-1}, G\right)\right) \rightarrow H^{n+1}\left(E_{n-1}, E_{A} ; \pi_{n}(G)\right) .
$$

We will write $\lambda_{\Lambda}^{n+1}(\xi)=\lambda^{n+1}(\xi) \in H^{n+1}\left(E_{n-1} ; \pi_{n}(G)\right)$ if $A$ is empty.

Proposition 5.6. The map $h_{n-1}:\left(E, E_{A}\right) \rightarrow\left(E_{n-1}, E_{A}\right)$ has the property

$$
h_{n-1}^{*}\left(\lambda_{A}^{n+1}(\xi)\right)=0 .
$$

Proof. Consider the commutative diagram

$$
\begin{gathered}
H^{n+1}\left(E_{n-1}, E ; \pi\right) \stackrel{j^{*}}{\longrightarrow} H^{n+1}\left(E_{n-1}, E_{A} ; \pi\right) \stackrel{h_{n-1}^{*}}{\longrightarrow} H^{n+1}\left(E, E_{A} ; \pi\right) \\
\downarrow \partial_{*} \\
H^{n+1}\left(E_{n-1}, E_{A} ; \pi_{n}(G)\right) \stackrel{j^{*}}{\longrightarrow} H^{n+1}\left(E_{n-1}, E_{A} ; \pi_{n}(G)\right) \stackrel{h_{n-1}^{*}}{\longrightarrow} H^{n+1}\left(E, E_{A} ; \pi_{n}(G)\right),
\end{gathered}
$$

where $\pi=\pi_{n+1}\left(G_{n-1}, G\right)$. Recall that by definition $\lambda^{n+1}\left(\xi_{n-1}, \xi\right) \in$ image $j^{*}$, and note that the horizontal lines are portions of the exact sequence of the triple $\left(E_{n-1}, E, E_{A}\right)$, whence 


$$
h_{n-1}^{*}\left(\lambda_{A}^{n+1}(\xi)\right)=h_{n-1}^{*} \partial_{*}\left(\lambda_{A}^{n+1}\left(\xi_{n-1}, \xi\right)\right)=\partial_{*} h_{n-1}^{*}\left(\lambda_{A}^{n+1}\left(\xi_{n-1}, \xi\right)\right)=0 .
$$

Observe that if $k: E_{n-1} \rightarrow\left(E_{n-1}, E_{A}\right)$ is the inclusion map, then

$$
k^{*}\left(\lambda_{A}^{n+1}(\xi)\right)=\lambda^{n+1}(\xi)
$$

Proposition 5.7. If $\xi$ admits a cross-section $f: B^{n} \cup A \rightarrow E$ and $f^{\prime}: B \rightarrow E_{n-1}$ is an extension of $h_{n-1} f: B^{n} \cup A \rightarrow E_{n-1}$, then

$$
c^{n+1}(f)=f^{*}\left(\lambda_{A}^{n+1}(\xi)\right) \in H^{n+1}\left(B, A ; \pi_{n}(G)\right) .
$$

Proof. This is just formula 3.5.

The pair $\left(G_{n-1}, G\right)$ is $(n+1)$-simple, and there is a fundamental class $\theta^{n+1}\left(G_{n-1}, G\right) \in H^{n+1}\left(G_{n-1}, G ; \pi_{n+1}\left(G_{n-1}, G\right)\right)$. Since $G \rightarrow G_{N} \rightarrow \cdots \rightarrow G_{1}$ is a (finite) Postnikov system for $G$, we may define the $(n+1)$ st Postnikov invariant $k^{n+1}(G) \in H^{n+1}\left(G_{n-1} ; \pi_{n}(G)\right)$ as the image of $\theta^{n+1}\left(G_{n-1}, G\right)$ under the composition

$$
B^{n+1}\left(G_{n-1}, G ; \pi\right) \stackrel{j^{\prime *}}{\rightarrow} H^{n+1}\left(G_{n-1} ; \pi\right) \stackrel{\partial_{*}}{\rightarrow} H^{n+1}\left(G_{n-1}, \pi_{n}(G)\right),
$$

where $\pi=\pi_{n+1}\left(G_{n-1}, G\right)$.

Proposition 5.8. If $i_{n-1}: G_{n-1} \rightarrow E_{n-1}$ is the inclusian of the fibre, then

$$
i_{n-1}^{*}\left(\lambda^{n+1}(\xi)\right)=k^{n+1}(G) \text {. }
$$

Proof. Consider the commutative diagram

$$
\begin{aligned}
& H^{n+1}\left(E_{n-1}, E ; \pi\right) \stackrel{j^{*}}{\rightarrow} B^{n+1}\left(E_{n-1} ; \pi\right) \stackrel{\partial_{*}}{\rightarrow} H^{n+1}\left(E_{n-1} ; \pi_{n}(G)\right)
\end{aligned}
$$

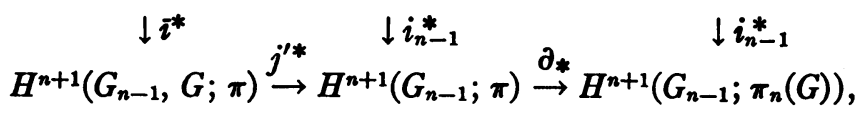

where $\pi=\pi_{n+1}\left(G_{n-1}, G\right)$, and recall that $\lambda^{n+1}(\xi)=\partial_{*} j^{*} i^{*-1}\left(\theta^{n+1}\right)$, where $\theta^{n+1}$ $=\theta^{n+1}\left(G_{n-1}, G\right)$. Thus, $i_{n-1}^{*}\left(\lambda^{n+1}(\xi)\right)=\partial_{*} i_{n-1}^{*} j^{*} i^{*-1}\left(\theta^{n+1}\right)=\partial_{*} j^{*} i_{i}^{*} i^{*-1}\left(\theta^{n+1}\right)$ $=\partial_{*} j^{*}\left(\theta^{n+1}\right)=k^{n+1}(G)$.

We have not investigated to what extent the invariants $\lambda^{n+1}(\xi)$ determine the bundle $\xi$. This is a problem for further research.

Proposition 5.9. If $\xi=(E, p, B, G, G)$ is a principal bundle, and $\phi: B^{\prime} \rightarrow B$ is a map, let $\left(\phi_{n-1}, \phi\right): \phi^{-1}\left(\xi_{n-1}\right) \rightarrow \xi_{n-1}$ be the induced bundle map. Then

$$
\phi_{n-1}^{*}\left(\lambda^{n+1}(\xi)\right)=\lambda^{n+1}\left(\phi^{-1}(\xi)\right) \in H^{n+1}\left(E_{n-1}^{\prime} ; \pi_{n}(G)\right) \text {. }
$$

Proof. If $\phi_{n-1}^{\prime}$ is the induced map $\left(E_{n-1}^{\prime}, E_{n}\right) \rightarrow\left(E_{n-1}, E_{n}\right)$, the proof follows very easily if we "chase around" the commutative diagram 


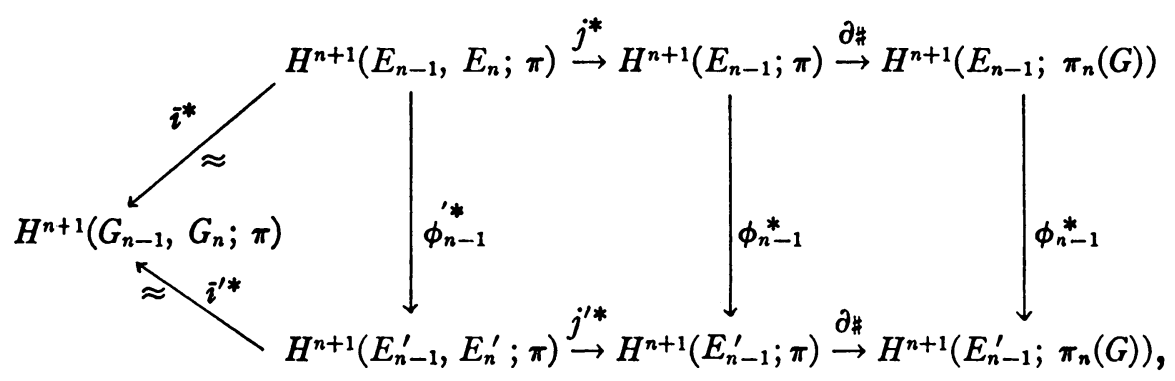

where $\pi=\pi_{n+1}\left(G_{n-1}, G_{n}\right)$.

Let $\left(\xi(G), N,\left\{\xi_{n}\right\},\left\{\left(\xi_{n}, 1, \gamma_{n}\right)\right\}\right)$ be a decomposition of a universal $G$ bundle $\xi(G)=\left(E_{G}, \pi, B_{G}, G, G\right)$. For notational purposes, let $\xi_{n}$ $=\left(\tilde{E}_{n}, \tilde{\pi}_{n}, B_{G}, G_{n}, G_{n}\right)$, and let $\pi=\pi_{n+2}\left(G_{n}, G_{n+1}\right) \approx \pi_{n+1}\left(G_{n+1}\right) \approx \pi_{n+1}(G)$. In the sections that follow, it will be useful to have the following facts.

PROPOSITION 5.10. (i) $\pi_{i}\left(\widetilde{E}_{n}\right)=0$ for $i<n+2, \pi_{i}\left(\tilde{E}_{n}\right) \approx \pi_{i-1}(G)$ for $i \geqq n+2$,

(ii) $H_{i}\left(\tilde{E}_{n}\right)=0$ for $i=1,2, \cdots, n+1$, and $H_{n+2}\left(\tilde{E}_{n}\right)=\pi$,

(iii) $H^{i}\left(\tilde{E}_{n}\right)=0$ for $i=1,2, \cdots, n+1$, and $H^{n+2}\left(E_{n} ; \pi\right)$ is a cyclic $\operatorname{Hom}(\pi, \pi)$ module and is generated by $\lambda^{n+2}(\xi(G))$.

Proof. From property (ii) of 5.3, $h_{n}^{*}: \pi_{i}\left(E_{G}\right) \rightarrow \pi_{i}\left(\tilde{E}_{n}\right)$ is onto for $i \leqq n+1$, and since $\pi_{i}\left(E_{G}\right)=0$ for all $i, \pi_{i}\left(E_{n}\right)=0$ for $i<n+2$. From the homotopy sequence of $\xi_{n}$, since $\pi_{i}\left(G_{n}\right)=0$ for $i>n, p_{n}^{*}: \pi_{i}\left(\tilde{E}_{n}\right) \rightarrow \pi_{i}\left(B_{G}\right)$ is an isomorphism onto for $i \geqq n+2$. But $\pi_{i}\left(B_{G}\right) \approx \pi_{i-1}(G)$, so $\pi_{i}\left(E_{n}\right) \approx \pi_{i-1}(G)$ for $i \geqq n+2$. (ii) follows from the Hurewicz theorem. By the universal coefficient theorem, $H^{i}\left(\tilde{E}_{n}\right)=0$ for $i=1,2, \cdots, n+1$, and $H^{n+2}\left(E_{n} ; \pi\right)=\operatorname{Hom}(\pi, \pi)$. To see that $\lambda^{n+2}(\xi(G))$ is a generator, observe that $H^{n+1}\left(E_{n+1} ; \pi\right)=H^{n+2}\left(\tilde{E}_{n+1} ; \pi\right)=0$, so that $j: \tilde{E}_{n} \rightarrow\left(\tilde{E}_{n}, \tilde{E}_{n+1}\right)$ induces an isomorphism onto, $j^{*}: H^{n+2}\left(\tilde{E}_{n}, \tilde{E}_{n+1} ; \pi\right)$ $\rightarrow H^{n+2}\left(\tilde{E}_{n} ; \pi\right)$, and that $\lambda^{n+2}(\xi(G))$ is the image of the generator of $H^{n+2}\left(G_{n}, G_{n+1} ; \pi\right)$ under the composition

$$
H^{n+2}\left(G_{n}, G_{n+1} ; \pi\right) \stackrel{i^{*-1}}{\longrightarrow} H^{n+2}\left(\tilde{E}_{n}, \tilde{E}_{n+1} ; \pi\right) \stackrel{j^{*}}{\longrightarrow} H^{n+2}\left(\tilde{E}_{n} ; \pi\right) .
$$

6. The obstruction theorems. The theorems of this section and their corollaries are the main results for a principal bundle $\xi$ whose fibre is an arbitrary connected countable $\mathrm{CW}$-group.

We remark that a necessary condition that a fibre space $\xi=(E, p, B, F)$ admit a cross-section $f: B^{n+1} \rightarrow E$ is that the homomorphism $p^{*}: H^{n+1}(B ; \pi)$ $\rightarrow H^{n+1}(E, \pi)$ be an isomorphism into. Thus if this necessary condition is satisfied, the formulae of 6.1 and 6.4 below completely determine the obstruction.

Theorem 6.1. Let $\xi=(E, p, B, G, G)$ be a principal bundle, let $\left(\xi, N,\left\{\xi_{m}\right\},\left\{\left(g_{m}, 1, \gamma_{m}\right)\right\}\right)$ be a decomposition of length $N \geqq n$, let $f: B^{n} \rightarrow E$ be 
a cross-section, and let $f^{\prime}: B \rightarrow E_{n-1}$ be an extension of $h_{n-1} f: B^{n} \rightarrow E_{n-1}$, so that $h_{f^{\prime}}: E_{n-1} \rightarrow G_{n-1}$ is defined. Then

$$
p^{*}\left(\bar{c}^{n+1}(f)\right)+h_{n-1}^{*} h_{f^{\prime}}^{*}\left(k^{n+1}(G)\right)=0 .
$$

Theorem 6.2. Let $\xi=(E, p, B, G, G)$ be a principal bundle, let $\left(\xi, N,\left\{\xi_{m}\right\},\left\{\left(g_{m}, 1, \gamma_{m}\right)\right\}\right)$ be a decomposition of length $N \geqq n$, let $f_{0}, f_{1}: B^{n} \rightarrow E$ be cross-sections, and let $f_{0}^{\prime}, f_{1}^{\prime}: B \rightarrow E_{n-1}$ be extensions of $h_{n-1} f_{0}$ and $h_{n-1} f_{1}$ so that $h_{f_{0} f_{1}}: B \rightarrow G_{n-1}$ is defined. Then

$$
\bar{c}^{n+1}\left(f_{1}\right)-\bar{c}^{n+1}\left(f_{0}\right)=h_{f^{\prime} \sigma^{\prime} 1}^{*}\left(k^{n+1}(G)\right) \text {. }
$$

This agrees with formula (30) of Dold and Whitney [7].

As an obvious corollary which has application later, we state the following.

CoRollary 6.3. If $k^{n+1}(G)=0$ and the $(n+1)$-dimensional obstruction is defined, then it is a single cohomology class.

Let $\xi=(E, p, B, G, G)$ be a principal bundle such that $\pi_{i}(G)=0$ for $0 \leqq i<n, n<i<q$. Then in the Postnikov system for $G$ we may take $G_{1}=\ldots$ $=G_{n-1}$ to be contractible groups, and $G_{n}=\cdots=G_{q-1}$ are $K\left(\pi_{n}(G), n\right)$ spaces. The $k$-invariant $k^{q+1}(G)=k^{q+1} \in H^{q+1}\left(G_{q-1} ; \pi_{q}(G)\right)$ is $k^{q+1} \vdash \theta^{n}\left(G_{q-1}\right)$, where $\theta^{n}\left(G_{q-1}\right)$ is the fundamental class, and $k^{q+1} \vdash$ is the cohomology operation defined by $k^{q+1}$. If $f: B^{q} \rightarrow E$ is a cross-section of $\xi$, then there is a unique class $\bar{a}^{n}(f) \in H^{n}\left(E ; \pi_{n}(G)\right)$ such that $i^{*}\left(\bar{a}^{n}(f)\right)=\theta^{n}(G)$ and $f^{*}\left(\bar{a}^{n}(f)\right)=0$, where $i: G \rightarrow E$ is the inclusion of the fibre.

Theorem 6.4. If $\xi=(E, p, B, G, G)$ is a principal bundle such that $\pi_{i}(G)=0$ for $0 \leqq i<n$ and $n<i<q$, and if $f: B^{q} \rightarrow E$ is a cross-section, then

$$
p^{*}\left(\bar{c}^{q+1}(f)\right)=k^{q+1} \vdash \tilde{a}^{n}(f) .
$$

TheOREM 6.5. If $\xi=(E, p, B, G, G)$ is a principal bundle such that $\pi_{i}(G)=0$ for $0 \leqq i<n$ and $n<i<q$, and if $f_{0}, f_{1}: B^{q} \rightarrow E$ are cross-sections, then

$$
\bar{c}^{q+1}\left(f_{1}\right)-\bar{c}^{q+1}\left(f_{0}\right)=k^{q+1} \vdash \bar{d}^{n}\left(f_{0} f_{1}\right) .
$$

CoRollary 6.6. If $\xi=(E, p, B, G, G)$ is a principal bundle such that $\pi_{i}(G)$ $=0$ for $0 \leqq i<n$ and $n<i<q$, and if $\xi$ admits a cross-section $f: B^{q} \rightarrow E$, then $\xi$ admits a cross-section $f^{\prime}: B^{q+1} \rightarrow E$ if and only if there is an element $d^{n}$ $\in H^{n}\left(B ; \pi_{n}(G)\right)$ such that

$$
\bar{c}^{q+1}(f)+k^{q+1} \vdash d^{n}=0 .
$$

In the case of a 3 -sphere bundle $\xi^{\prime}=\left(E^{\prime}, p^{\prime}, B, S^{3}, S O(4)\right)$ which admits cross-sections $f_{0}, f_{1}: B^{4} \rightarrow E^{\prime}$, Liao [12] obtains the formulae

$$
\begin{aligned}
p^{\prime *}\left(\bar{c}^{5}\left(f_{0}\right)\right) & =p^{\prime *}\left(w_{2}\left(\xi^{\prime}\right)\right) \cdot \bar{a}^{8}\left(f_{0}\right)+S q^{2} \bar{a}^{3}\left(f_{0}\right), \\
\bar{c}^{5}\left(f_{1}\right)-\bar{c}^{b}\left(f_{0}\right) & =w_{2}\left(\xi^{\prime}\right) \cdot \bar{d}^{3}\left(f_{0}, f_{1}\right)+S q^{2} \bar{d}^{3}\left(f_{0}, f_{1}\right),
\end{aligned}
$$


where $w_{2}\left(\xi^{\prime}\right)$ is the Stiefel-Whitney class, and $\bar{a}^{3}\left(f_{0}\right)$ is as above. Recall that $S^{z}=S p(1) \subset U(2) \subset S O(4)$, hence there exist maps $B_{S_{p(1)} \rightarrow B_{U(2)} \rightarrow B_{S O(4)}}$. The bundle $\xi^{\prime}$ is induced by a map $\phi: B \rightarrow B_{\text {so( }}$ and the associated sphere bundle $\left(B_{S O(3)}, \bar{p}, B_{S O(4)}, S^{3}, S O(4)\right)$, and if we can reduce the group of $\xi^{\prime}$ so as to get a principal $S^{3}$-bundle, the map $\phi$ factors through $B_{S p(1)}$, hence $w_{2}\left(\xi^{\prime}\right)=0$. But then Liao's formulae would be

$$
\begin{aligned}
p^{*}\left(\bar{c}^{b}(f)\right) & =S q^{2} \bar{a}^{8}(f), \\
\bar{c}^{5}\left(f_{1}\right)-\bar{c}^{b}\left(f_{0}\right) & =S q^{2} \bar{d}^{3}\left(f_{0}, f_{1}\right),
\end{aligned}
$$

which are precisely the formulae that result from 6.4 and 6.5.

Before we proceed with the proofs we will need some notation and a lemma. Let $\xi=(E, p, B, G, G)$ be a principal bundle, let $\xi=\left(E_{G}, \tilde{p}, B_{G}, G, G\right)$ be a universal bundle, and let $\phi_{\xi}: B \rightarrow B_{G}$ be a classifying map for $\xi$. Then a decomposition $\left(\xi, N,\left\{\xi_{m}\right\},\left\{\left(g_{m}, 1, \gamma_{m}\right)\right\}\right)$ is induced by a decomposition $\left(\xi, N,\left\{\xi_{m}\right\},\left\{\left(\tilde{\xi}_{m}, 1, \gamma_{m}\right)\right\}\right)$ of the universal bundle $\xi$. Let $\left(\phi_{\xi}^{m}, \phi_{\xi}, 1\right): \xi_{m} \rightarrow \tilde{\xi}_{m}$ be the induced bundle homomorphism. Then by Proposition 5.9, $\lambda^{n+1}(\xi)$ $=\phi^{n-1 *}\left(\lambda^{n+1}(\xi)\right) \in H^{n+1}\left(E_{n-1} ; \pi_{n}(G)\right)$.

LEMMA 6.7. If $\mu_{n-1}: G_{n-1} \times E_{n-1} \rightarrow E_{n-1}$ is the operation of $G_{n-1}$ on $E_{n-1}$, then $\mu_{n-1}^{*}\left(\lambda^{n+1}(\xi)\right)=p_{1}^{*}\left(k^{n+1}(G)\right)+p_{2}^{*}\left(\lambda^{n+1}(\xi)\right)$.

Proof. By (ii) of 5.10, $H_{r}\left(\tilde{E}_{n-1}\right)=0$ for $0<r<n+1$. Using this fact and the Künneth formula

$$
\begin{aligned}
H_{q}\left(G_{n-1} \times \tilde{E}_{n-1}, G_{n-1} \bigvee \tilde{E}_{n-1}\right) & \approx \sum_{r} H_{q-r}\left(G_{n-1}, e\right) \otimes H_{r}\left(\tilde{E}_{n-1}, x_{0}\right) \\
& +\sum_{r} \operatorname{Tor}\left(H_{q-r-1}\left(G_{n-1}, e\right), H_{r}\left(\tilde{E}_{n-1}, x_{0}\right)\right),
\end{aligned}
$$

we establish that $H_{q}\left(G_{n-1} \times \tilde{E}_{n-1}, G_{n-1} \bigvee \tilde{E}_{n-1}\right)=0$, for $q \leqq n+1$. From the universal coefficient theorem, we find

$$
H^{q}\left(G_{n-1} \times \widetilde{E}_{n-1}, G_{n-1} \vee \tilde{E}_{n-1} ; \pi\right)=0,
$$

for $q \leqq n+1$ and any abelian group $\pi$. Consider the commutative diagram

$$
\begin{aligned}
& H^{n+1}\left(\tilde{E}_{n-1} ; \pi\right) \stackrel{\tilde{\mu}_{n-1}^{*}}{\longrightarrow} H^{n+1}\left(G_{n-1} \times \tilde{E}_{n-1} ; \pi\right) \\
& \begin{array}{c}
\downarrow \phi_{\xi}^{n-1 *} \\
\downarrow H^{n+1}\left(E_{n-1} ; \pi\right) \stackrel{\mu_{n-1}^{*}}{\longrightarrow} H^{n+1}\left(G_{n-1} \times \phi_{\xi-1}^{n-1}\right)^{*} \\
\left.E_{n}\right) .
\end{array} \\
& \mu_{n-1}^{*}\left(\lambda^{n+1}(\xi)\right)=\mu_{n-1}^{*} \phi_{\xi}^{n-1 *}\left(\lambda^{n+1 *}(\xi)\right)=\left(1 \times \phi_{\xi}^{n-1}\right)^{*} \tilde{\mu}_{n-1}^{*}\left(\lambda^{n+1}(\xi)\right) \\
& =\left(1 \times \phi_{\xi}^{n-1}\right)^{*}\left(p_{1}^{*}\left(k^{n+1}(G)\right)+p_{2}^{*}\left(\lambda^{n+1}(\xi)\right)\right),
\end{aligned}
$$

by 4.10 and the fact that $H^{n+1}\left(G_{n-1} \times E_{n-1}, G_{n-1} \bigvee E_{n-1} ; \pi\right)=0$. By 4.9, $\mu_{n-1}^{*}\left(\lambda^{n+1}(\xi)\right)=p_{1}^{*}\left(k^{n+1}(G)\right)+p_{2}^{*} \phi_{\xi}^{n-1 *}\left(\lambda^{n+1}(\xi)\right)=p_{1}^{*}\left(k^{n+1}(G)\right)+p_{2}^{*}\left(\lambda^{n+1}(\xi)\right)$. 
Proof of 6.1. It is easily checked that the composition

$$
E_{n-1} \stackrel{\Delta}{\rightarrow} E_{n-1} \times E_{n-1} \stackrel{\alpha}{\rightarrow} G_{n-1} \times B \stackrel{\beta}{\rightarrow} G_{n-1} \times E_{n-1} \stackrel{\mu_{n-1}}{\rightarrow} E_{n-1}
$$

is the identity map on $E_{n-1}$ where $\alpha=h_{f^{\prime}} \times p_{n-1}$ and $\beta=1 \times f^{\prime}$. Thus

$$
\begin{aligned}
\lambda^{n+1}(\xi) & =\Delta^{*}\left(h_{f^{\prime}} \times p_{n-1}\right)^{*}\left(1 \times f^{\prime}\right)^{*} \mu_{n-1}^{*}\left(\lambda^{n+1}(\xi)\right) \\
& =\Delta^{*}\left(h_{f^{\prime}} \times p_{n-1}\right)^{*}\left(1 \times f^{\prime}\right)^{*}\left(p_{1}^{*}\left(k^{n+1}(G)\right)+p_{2}^{*}\left(\lambda^{n+1}(\xi)\right)\right) \\
& =h_{f^{\prime}}^{*}\left(k^{n+1}(G)\right)+p_{n-1}^{*}\left(c^{n+1}(f)\right),
\end{aligned}
$$

by 5.7 . By 5.6 , the map $h_{n-1}: E \rightarrow E_{n-1}$ has the property $h_{n-1}^{*}\left(\lambda^{n+1}(\xi)\right)=0$, whence

$$
\begin{aligned}
0=h_{n-1}^{*}\left(\lambda^{n+1}(\xi)\right) & =h_{n-1}^{*} h_{f^{\prime}}^{*}\left(k^{n+1}(G)\right)+h_{n-1}^{*} p_{n-1}^{*}\left(\bar{c}^{n+1}(f)\right) \\
& =h_{n-1}^{*} h_{f^{\prime}}^{*}\left(k^{n+1}(G)\right)+p^{*}\left(\bar{c}^{n+1}(f)\right) .
\end{aligned}
$$
Thus,

Proof of 6.2. As above, we find that $\lambda^{n+1}(\xi)=p_{n-1}^{*}\left(\bar{c}^{n+1}\left(f_{0}\right)\right)+h_{f_{0}^{\prime}}^{\prime}\left(k^{n+1}(G)\right)$.

$$
\begin{aligned}
\bar{c}^{n+1}\left(f_{1}\right) & =f_{1}^{\prime *}\left(\lambda^{n+1}(\xi)\right)=f_{1}^{\prime *} p_{n-1}^{*}\left(\bar{c}^{n+1}\left(f_{0}\right)\right)+f_{1}^{\prime *} h_{f_{0}^{\prime}}^{*}\left(k^{n+1}(G)\right) \\
& =\bar{c}^{n+1}\left(f_{0}\right)+h_{f_{j}^{\prime} f_{1}^{\prime}}^{*}\left(k^{n+1}(G)\right) .
\end{aligned}
$$

Transposing $\bar{c}^{n+1}\left(f_{0}\right)$, we obtain the desired formula.

Proof of 6.4. Since $\pi_{i}(G)=0$ for $0 \leqq i<n$ and $n<i<q, G_{q-1}$ has the homotopy type of $G_{n}$, which is a $K\left(\pi_{n}(G), n\right)$. Thus $k^{q+1}(G) \in H^{q+1}\left(G_{q-1} ; \pi_{q}(G)\right)$ determines an operation

$$
k^{q+1} \vdash: H^{n}\left(G_{q-1} ; \pi_{n}(G)\right) \rightarrow H^{q+1}\left(G_{q-1} ; \pi_{q}(G)\right),
$$

i.e., $k^{q+1}(G)=k^{q+1} \vdash \theta^{n}\left(G_{q-1}\right)$. Since $f: B^{q} \rightarrow E$ is a cross-section, $h_{f}: p^{-1}\left(B^{q}\right) \rightarrow G$ is defined. Consider the diagram

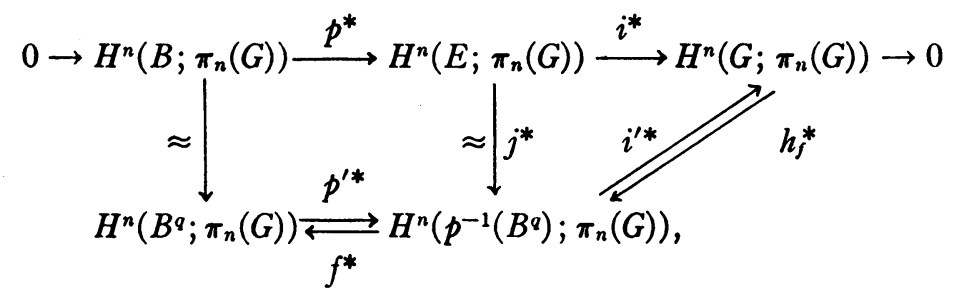

where the horizontal line is a portion of Serre's exact sequence, and $j: p^{-1}\left(B^{q}\right)$ $\rightarrow E$ is the inclusion. Since the inclusion $i^{\prime}: p^{-1}\left(b_{0}\right) \rightarrow E$ may be given as $i^{\prime}(g)=g \cdot f\left(b_{0}\right)$, we have $h_{f} i^{\prime}(g)=h_{f}\left(g \cdot f\left(b_{0}\right)\right)=g \cdot e=g$, so if $\bar{a}^{n}(f) \in H^{n}\left(E ; \pi_{n}(G)\right)$ is such that $i^{*}\left(\bar{a}^{n}(f)\right)=\theta^{n}(G)$ and $f^{*}\left(\bar{a}^{n}(f)\right)=0, j^{*}\left(\bar{a}^{n}(f)\right)=h_{f}^{*}\left(\theta^{n}(G)\right)$. By 6.1,

$$
0=p^{*}\left(\bar{c}^{q+1}(f)\right)+h_{q-1}^{*} h_{f^{\prime}}^{*}\left(k^{q+1} \vdash \theta^{n}\right)=p^{*}\left(\bar{c}^{q+1}(f)\right)+k^{q+1} \vdash h_{q-1}^{*} h_{f^{\prime}}^{*}\left(\theta^{n}\right),
$$


where $\theta^{n}=\theta^{n}\left(G_{q-1}\right)$. But commutativity of the diagram

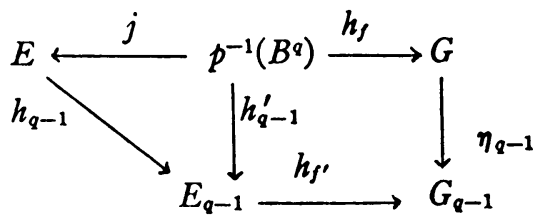

follows from 4.3 since $\left(h_{q-1}, 1, \eta_{q-1}\right): \xi \rightarrow \xi_{q-1}$ is a homomorphism of principal fibre spaces, and implies that

$$
j^{*} h_{q-1}^{*} h_{f^{\prime}}^{*}\left(\theta^{n}\left(G_{q-1}\right)\right)=h_{f}^{*} \eta_{q-1}^{*}\left(\theta^{n}\left(G_{q-1}\right)\right)=h_{f}^{*}\left(\theta^{n}(G)\right)=j^{*}\left(\tilde{a}^{n}(f)\right) .
$$

Thus $h_{q-1}^{*} h_{f^{\prime}}^{*}\left(\theta^{n}\left(G_{q-1}\right)\right)=\bar{a}^{n}(f)$ because $j^{*}$ is an isomorphism, and

$$
0=p^{*}\left(\bar{c}^{q+1}(f)\right)+k^{q+1} \vdash \bar{a}^{n}(f) .
$$

Proof of 6.5. By 6.2,

$$
\bar{c}^{q+1}\left(f_{1}\right)-\bar{c}^{q+1}\left(f_{0}\right)=h_{f^{\prime} f_{1}^{\prime}}^{*}\left(k^{q+1}(G)\right)=k^{q+1} \vdash h_{f_{0}^{\prime} f_{1}^{\prime}}^{*}\left(\theta^{n}\left(G_{q-1}\right)\right) .
$$

But $\eta_{q-1} h_{f_{0} f_{1}}=\eta_{q-1} h_{f_{1}} f_{0}=h_{f_{0}}^{\prime} h_{q-1} f_{1}=h_{f_{\sigma_{1}}^{\prime}}^{\prime} \mid B^{a}$ by 4.3 , so

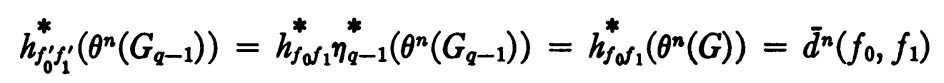

by 4.6. Hence,

$$
\bar{c}^{q+1}\left(f_{1}\right)-\bar{c}^{q+1}\left(f_{0}\right)=k^{q+1} \vdash \bar{d}^{n}\left(f_{0}, f_{1}\right) .
$$

Proof of 6.6. If $\xi$ admits a cross-section $f^{\prime}: B^{a+1} \rightarrow E$, then $0=\bar{c}^{q+1}\left(f^{\prime}\right)$ $=k^{q+1} \vdash \bar{d}^{n}\left(f, f^{\prime}\right)+\bar{c}^{q+1}(f)$. If $\bar{c}^{q+1}(f)+k^{q+1} \vdash d^{n}=0$, choose $f^{\prime}: B^{q} \rightarrow E$ such that $\bar{d}^{n}\left(f, f^{\prime}\right)=d^{n}$. Then $\bar{c}^{q+1}\left(f^{\prime}\right)=0$, and $f^{\prime} \mid B^{q-1}$ extends over $B^{q+1}$.

7. Principal bundles with fibre $U(n)\left({ }^{2}\right)$. We need some results on the homotopy groups of $U(n)$, namely those of Bott [5], that $\pi_{i}(U(n))=Z$ for $i \equiv 1(\bmod 2), i<2 n, \pi_{i}(U(n))=0$ for $i \equiv 0(\bmod 2), i<2 n$, and $\pi_{2 n}(U(n))$ $=Z_{n !}$. We also need the fact that $H^{*}\left(B_{U(n)}\right)=Z\left[\tilde{\tau}_{1}, \cdots, \tilde{z}_{n}\right]$, an integral polynomial ring with generators $\tilde{z}_{i}$ of degree $2 i$. The class $\boldsymbol{\tau}_{i}$ is the $i$ th universal Chern class. If $\xi=(E, p, B, U(n), U(n))$ is a principal bundle and $\phi_{\xi}: B \rightarrow B_{U(n)}$ is a classifying map, then $\phi_{\xi}^{*}\left(\tilde{c}_{i}\right)=c_{i}(\xi)$ is the $i$ th Chern class of $\xi$.

We propose to calculate the relationship between the Chern classes $c_{i}(\xi)$ and the obstructions to a cross-section of the bundle $\xi$. At first we will work in the universal bundle $\xi=\left(E_{U(n)}, \tilde{p}, B_{U(n)}, U(n), U(n)\right)$, and we will always assume that we have a decomposition $\left(\xi, N,\left\{\xi_{m}\right\},\left\{\left(\xi_{m}, 1, \gamma_{m}\right)\right\}\right)$ of length $N \geqq 2 n$ (for $U(n)$-bundles). For notation we write $\xi_{m}=\left(\tilde{E}_{m}, \tilde{p}_{m}, B_{U(n)}, G_{m}, G_{m}\right)$.

THEOREM 7.1. Let $\lambda^{2 k}(\tilde{\xi}) \in H^{2 k}\left(\tilde{E}_{2 k-2} ; \pi_{2 k-1}(U(n))\right)=H^{2 k}\left(\tilde{E}_{2 k-2}\right)$ for $n \geqq k$, be

$\left({ }^{2}\right)$ We actually consider a bundle whose fibre is a connected CW-group having the same homotopy type as $U(n)$, but in order to simplify the notation, we denote this group by $U(n)$. This convention will be followed in 888 and 9. See the remark following 5.2. 
as previously defined. Then

$$
\tilde{p}_{2 k-2}^{*}\left(\tilde{c}_{k}\right)= \pm(k-1) ! \lambda^{2 k}(\xi) .
$$

Proof. Let $S^{2 k}$ be a $(2 k)$-sphere, and choose a map $\phi: S^{2 k} \rightarrow B_{U(n)}$ which represents a generator of $\pi_{2 k}\left(B_{U(n)}\right)=Z$. Then the induced map $\phi_{*}: \pi_{2 k}\left(S^{2 k}\right)$ $\rightarrow \pi_{2 k}\left(B_{U(n)}\right)$ is an isomorphism onto. The obstructions to lifting $\phi$ to a map $\phi^{\prime}: S^{2 k} \rightarrow \tilde{E}_{2 k-2}$ are elements of the group $H^{i}\left(S^{2 k}, \pi_{i-1}\left(G_{2 k-2}\right)\right)=0$, so such a lifting exists, i.e., there is a commutative diagram

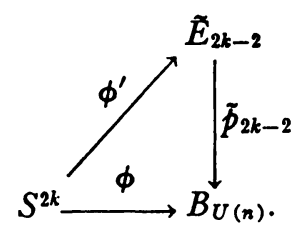

Since $\phi_{*}: \pi_{2 k}\left(S^{2 k}\right) \rightarrow \pi_{2 k}\left(B_{U(n)}\right)$ and $\tilde{p}_{2 k-2 *}: \pi_{2 k}\left(\tilde{E}_{2 k-2}\right) \rightarrow \pi_{2 k}\left(B_{U(n)}\right)$ are isomorphisms onto, and since $\pi_{i}\left(S^{2 k}\right)=\pi_{i}\left(\tilde{E}_{2 k-2}\right)=0$ for $i<2 k$, it follows that $\phi_{*}^{\prime}: \pi_{i}\left(S^{2 k}\right) \rightarrow \pi_{i}\left(\widetilde{E}_{2 k-2}\right)$ is an isomorphism onto for $i \leqq 2 k$. Thus $\phi^{\prime *}: H^{2 k}\left(\widetilde{E}_{2 k-2}\right)$ $\rightarrow H^{2 k}\left(S^{2 k}\right)$ is an isomorphism onto, and if $u \in H^{2 k}\left(S^{2 k}\right)$ is a generator, then $\phi^{\prime *}\left(\lambda^{n+1}(\tilde{\xi})\right)= \pm u$, by 5.10 .

We now wish to compute the obstruction to a cross-section of the bundle $\phi^{-1}(\xi)$. In order to do this, we prove the following general lemma about principal bundles over spheres which has applications in $\$ \$ 8$ and 9 as well as in the proof of this theorem. Thus let $\xi=\left(E, p, S^{n}, G, G\right)$ be a principal bundle with $\phi_{\xi}: S^{n} \rightarrow B_{G}$ as classifying map. Let $E_{+}^{n}$ and $E_{-}^{n}$ be the closed hemispheres of $S^{n}$ with $E_{+}^{n} \cap E_{-}^{n}=S^{n-1}$ as equator. Let an orientation of $S^{n}$ be given. We may assume that the classifying map has the property $\phi_{\xi}\left(E_{+}^{n}\right)=x_{0} \in B_{G}$, and that there is a cross-section $\Phi: E_{+}^{n} \rightarrow E$.

Leмma. The obstruction cocycle $c^{n}(\Phi)$ has the properties $c^{n}(\Phi)\left(E_{+}^{n}\right)=0$, $c^{n}(\Phi)\left(E_{-}^{n}\right)= \pm \partial\left(\left\{\phi_{\xi}\right\}\right)$, where $\partial$ is the boundary homomorphism in the homotopy sequence of $\xi$, and $\left\{\phi_{\xi}\right\}$ is the homotopy class of the map $\phi_{\xi}: S^{n} \rightarrow B G$.

Proof. The cross-section $\Phi: E_{+}^{n} \rightarrow E$ determines a lifting $\Phi^{\prime}: E_{+}^{n} \rightarrow E_{G}$ of $\phi_{\xi} \mid E_{+}^{n}$, i.e., $p \Phi^{\prime}=\phi_{\xi} \mid E_{+}^{n}$, and in particular, we have $p \Phi^{\prime}\left(S^{n-1}\right)=x_{0} \in B_{G}$. Thus $\Phi^{\prime}\left|\dot{E}_{+}^{n}=\Phi^{\prime}\right| S^{n-1}$ determines a map $S^{n-1} \rightarrow G$, and it is easily seen from the definition that $c^{n}(\Phi)\left(E_{-}^{n}\right)=\left\{\Phi^{\prime} \mid \dot{E}_{+}^{n}\right\} \in \pi_{n-1}(G)$. Now consider the commutative diagram

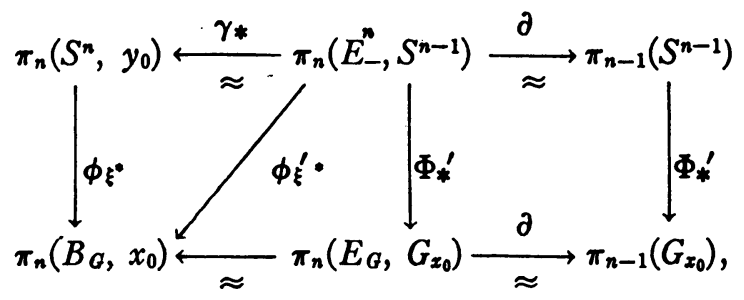


where $\gamma:\left(E_{-}^{n}, S^{n-1}\right) \rightarrow\left(S^{n}, y_{0}\right)$ is of degree one. Let $j_{n} \in \pi_{n}\left(E_{-}^{n}, S^{n-1}\right)$ be a generator. Then

$$
\begin{aligned}
c^{n}(\Phi)\left(E_{-}^{n}\right) & =\left\{\Phi^{\prime} \mid E_{-}^{n}\right\}= \pm \Phi_{*}^{\prime} \partial j_{n}= \pm \partial \Phi_{*}^{\prime} j_{n}= \pm \partial \tilde{p}_{*}^{-1} \tilde{p}_{*} \Phi_{*}^{\prime} j_{n}= \pm \partial \phi_{\xi *}^{\prime} j_{n} \\
& \left.= \pm \partial \phi_{\xi *}\left(i_{n}\right)= \pm \partial\left(\left\{\phi_{\xi}\right)\right\}\right) .
\end{aligned}
$$

Returning to the proof of the theorem, observe that since $\phi: S^{2 k} \rightarrow B_{U(n)}$ represents a generator of $\pi_{2 k}\left(B_{U(n)}\right)=Z$, the obstruction to a cross-section of $\phi^{-1}(\xi)$ is $\pm u \in H^{2 k}\left(S^{2 k}\right)$. Thus according to Kérvaire [10], $\phi^{*}\left(\tilde{z}_{k}\right)= \pm(k-1) ! u$ $= \pm(k-1) ! \phi^{\prime *}\left(\lambda^{2 k}(\xi)\right)$. Since $\phi^{*}\left(\tilde{\tau}_{k}\right)=\phi^{* *} \tilde{p}_{2 k-2}^{*}\left(\tilde{z}_{k}\right), \phi^{\prime *}\left(\tilde{p}_{2 k-2}^{*}\left(\mathcal{Z}_{k}\right) \mp(k-1) ! \lambda^{2 k}(\xi)\right)$ $=0$, and since $\phi^{*}$ is an isomorphism in dimension $2 k$,

$$
\tilde{p}_{2 k-2}^{*}\left(\tilde{c}_{k}\right)= \pm(k-1) ! \lambda^{2 k}(\xi) \text {. }
$$

CoRollaRy 7.2. Let $\xi=(E, p, B, U(n), U(n))$ be a principal bundle which admits a cross-section $f: B^{2 k-1} \rightarrow E$ for $n \geqq k$. Then

$$
c_{k}(\xi)= \pm(k-1) ! \bar{c}^{2 k}(f) \text {. }
$$

Proof. Consider the commutative diagram

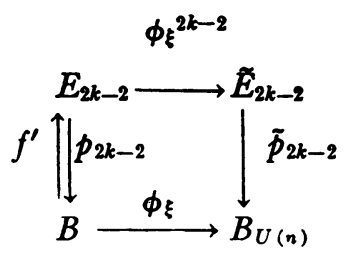

where $\phi_{\xi}$ is the classifying map for $\xi$. Then

$$
c_{k}(\xi)=\phi^{*}\left(\tilde{z}_{k}\right)=f^{\prime *} \phi^{2 k-2 *} p_{2 k-2}^{*}\left(\tilde{C}_{k}\right)=f^{*}\left( \pm(k-1) ! \lambda^{2 k}(\xi)\right)= \pm(k-1) ! \bar{c}^{2 k}(f) .
$$

Corollary 7.3. If $M^{2 n}$ is an almost complex manifold such that the cohomology torsion coefficients in dimension $2 k$ are relatively prime to $(k-1)$ !, $k=1,2, \cdots, n$, then $M^{2 n}$ is parallelizable if and only if all the Chern classes of its tangent bundle vanish.

This result agrees with that of Peterson [15].

Lemma 7.4. The class $k^{4}(U(n)) \in H^{4}\left(G_{2} ; \pi_{3}(U(n))\right)=H^{4}\left(G_{2}\right)$ is zero.

Proof. Since $\pi_{2}(U(n))=0, G_{2}=G_{1}$ which is a $K(Z, 1)$, so $H^{4}\left(G_{2}\right)=0$.

We remark that by Corollary 6.3 , this lemma implies that the secondary obstruction of a principal $U(n)$-bundle is a single cohomology class. This lemma also implies that $G_{3}$ has the same homotopy type as $K(Z, 1) \times K(Z, 3)$ (but not necessarily the group structure of a product).

Lemma 7.5. (i) $k^{5}(U(2))=S q^{2} \theta_{3} \in H^{5}\left(G_{3} ; \pi_{4}(U(2))\right)=H^{5}\left(G_{3} ; Z_{2}\right)$, where $\theta_{3}$ is a generator of $H^{3}\left(G_{3}\right)=Z$, 
(ii) $k^{6}(U(n))=S q^{3} \theta_{3} \in H^{0}\left(G_{4} ; \pi_{6}(U(n))\right)=H^{0}\left(G_{4}\right)$ for $n>2$.

Proof of (i). Consider the diagram

$$
\begin{aligned}
& 0 \rightarrow H^{4}\left(G_{3} ; Z_{2}\right) \stackrel{\gamma_{3}^{*}}{\rightarrow} H^{4}\left(G_{4} ; Z_{2}\right) \stackrel{\delta^{*}}{\rightarrow} H^{5}\left(G_{3}, G_{4} ; Z_{2}\right) \stackrel{j^{*}}{\rightarrow} H^{5}\left(G_{3} ; Z_{2}\right) \stackrel{\gamma_{3}^{*}}{\rightarrow} H^{5}\left(G_{4} ; Z_{2}\right) \\
& \eta_{3}^{*} \quad / \eta_{4}^{*} \\
& H^{4}\left(U(2) ; Z_{2}\right)
\end{aligned}
$$

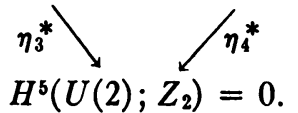

$H^{4}\left(G_{3} ; Z_{2}\right)$ is generated by the product of the 1-dimensional and the 3dimensional generators, as is $H^{4}\left(U(n) ; Z_{2}\right)$, hence $\eta_{3}^{*}$ is an isomorphism onto in dimension 4. Since $\eta_{4}^{*}$ is an isomorphism onto in dimension 4 , it follows that $\gamma_{3}^{*}: H^{4}\left(G_{3} ; Z_{2}\right) \rightarrow H^{4}\left(G_{4} ; Z_{2}\right)$ is an isomorphism onto, and $\gamma^{*}$ is the zero homomorphism. $H^{3}\left(G_{3} ; Z_{2}\right)$ is generated by $S q^{2} \theta_{3}$, so $j^{*}$ is an isomorphism onto, and $k^{5}(U(2))=S q^{2} \theta_{3}$.

The proof of (ii) is analogous, except that since $\pi_{4}(U(n))=0$, when $n>2$, we have $G_{3}=G_{4}$, so we use the cohomology sequence of the pair $\left(G_{4}, G_{6}\right)$ with integral coefficients.

We now compute the first three obstructions to a cross-section of a principal bundle $\xi=(E, p, B, U(n), U(n))$. It is clear that the primary obstruction to a cross-section, $\bar{c}^{2}(f) \in H^{2}(B)$, is the Chern class $c_{1}(\xi)$. If $n=1$, this is the only obstruction, because $U(1)=S^{1}$. Assume $n>1$. Then if $c_{1}(\xi)=0$, there is a cross-section $f: B^{3} \rightarrow E$, and by Corollary 7.2, $\bar{c}^{4}(f)=c_{2}(\xi) \in H^{4}(B)$.

Now let $u_{1} \in H^{1}(U(n))$ and $u_{3} \in H^{3}(U(n))$ be generators such that if $\eta_{3}^{*}: H^{i}\left(G_{3}\right) \rightarrow H^{i}(U(n))$ is the homomorphism induced by $\eta_{3}: U(n) \rightarrow G_{3}$, then $\eta_{3}^{*}\left(\theta_{1}\right)=u_{1}$ and $\eta_{3}^{*}\left(\theta_{3}\right)=u_{3}$. Suppose $c_{1}(\xi)=c_{2}(\xi)=0$ so that there is a crosssection $f_{0}: B^{4} \rightarrow E\left(f_{0}: B^{5} \rightarrow E\right.$ if $\left.n>2\right)$. Then if $f_{1}: B^{4} \rightarrow E\left(f_{1}: B^{5} \rightarrow E\right)$ is a crosssection and $f_{0}^{\prime}, f_{1}^{\prime}: B \rightarrow E_{3}\left(f_{0}^{\prime}, f_{1}^{\prime}: B \rightarrow E_{4}\right)$ are extensions of $h_{3} f_{0}$ and $h_{3} f_{1}$ $\left(h_{4} f_{0}\right.$ and $\left.h_{4} f_{1}\right)$, we have the following formulae:

(i) if $n=2$,

$$
\begin{aligned}
\bar{c}^{5}\left(f_{1}\right)-\bar{c}^{5}\left(f_{0}\right) & =h_{f_{0}^{\prime} f_{1}^{\prime}}^{*}\left(k^{5}(U(n))\right)=i_{f^{\prime} f_{1}^{\prime}}^{*}\left(S q^{2} \theta_{3}\right)=S q^{2} h_{f_{0}^{\prime} f_{1}^{\prime}}^{*}\left(\theta_{3}\right) \\
& =S q^{2} h_{f_{0} f_{1}}^{*} \eta_{3}^{*}\left(\theta_{3}\right)=S q^{2} h_{f_{0} f_{1}}^{*}\left(u_{3}\right)=S q^{2} \bar{\gamma}^{-8}\left(f_{0}, f_{1}\right),
\end{aligned}
$$

(ii) if $n>2$,

$$
\bar{c}^{6}\left(f_{1}\right)-\bar{c}^{6}\left(f_{0}\right)=S q^{3}\left(\bar{\gamma}^{3}\left(f_{0}, f_{1}\right) \quad\right. \text { (by a similar computation), }
$$

where $\bar{\gamma}^{3}\left(f_{0}, f_{1}\right)=h_{f_{\sigma_{1}}}^{*}\left(u_{3}\right)$. To complete our calculations, we need the following proposition.

Proposition 7.6. Let $\xi=(E, p, B, U(n), U(n))$ be a principal bundle which admits a cross-section $f_{0}: B^{4} \rightarrow E$. Then if $d \in H^{1}(B)$ and $\gamma \in H^{3}(B)$, there is a cross-section $f_{1}: B^{4} \rightarrow E$ such that $\bar{d}^{1}\left(f_{0}, f_{1}\right)=d$ and $\bar{\gamma}^{3}\left(f_{0}, f_{1}\right)=\gamma$. 
Proof. Let $f_{0}^{\prime}: B \rightarrow E_{3}$ be an extension of $h_{3} f_{0}$. Since $G_{3}$ has the homotopy type of $K(Z, 1) \times K(Z, 3)$, the homotopy classes of maps $h: B \rightarrow G_{3}$ are in oneto-one correspondence with the (cartesian product) $H^{1}(B) \times H^{3}(B)$, the correspondence being $\{h\} \leftrightarrow\left(h^{*} \theta_{1}, h^{*} \theta_{3}\right)$ where $\{h\}$ is the homotopy class of $h$. Choose a map $h: B \rightarrow G_{3}$ such that $h^{*} \theta_{1}=d$ and $h^{*} \theta_{3}=\gamma$, and let $f_{1}^{\prime}: B \rightarrow E_{3}$ be the cross-section $f_{1}^{\prime}(x)=h(x) \cdot f_{0}^{\prime}(x) . f_{1}^{\prime}$ defines a cross-section $f_{1}: B^{4} \rightarrow E$, so $h_{f_{0} f_{1}}: B \rightarrow U(n)$ is defined and $f_{1}(x)=h_{f_{0} f_{1}}(x) \cdot f_{0}(x)$. Now $h_{3} f_{1}(x)=\eta_{3} h_{f_{0} f_{1}}(x)$ - $h_{3} f_{0}(x)=h_{f_{0} h_{2} f_{1}}(x) \cdot f_{0}^{\prime}(x)$, so $h_{f_{0}^{\prime} h_{2} f_{1}}=\eta_{3} h_{f_{0} f_{1}}$. Since $h_{3} f_{1} \cong f_{1}^{\prime}\left|B^{4}, \eta_{3} h_{f_{0} f_{1}} \cong h_{f_{0}^{\prime} f_{1}^{\prime}}\right| B^{4}$ $=h \mid B^{4}$. Thus $\gamma=h^{*}\left(\theta_{3}\right)=h_{f_{0} f_{1}}^{*} \eta_{3}^{*}\left(\theta_{3}\right)=h_{f_{0} j_{1}}^{*}\left(u_{3}\right)=\bar{\gamma}^{3}\left(f_{0}, f_{1}\right)$, and $d=h^{*}\left(\theta_{1}\right)$ $=h_{f_{0} f_{1}}^{*}\left(u_{1}\right)=\bar{d}^{1}\left(f_{0}, f_{1}\right)$.

We summarize our calculations in the following theorem.

Theorem 7.7. Let $\xi=(E, p, B, U(n), U(n))$ be a principal bundle. Then

(i) the primary obstruction is $c_{1}(\xi)$,

(ii) if $c_{1}(\xi)=0$, the secondary obstruction is $c_{2}(\xi)$,

(iii) if $c_{1}(\xi)=c_{2}(\xi)=0$ and $f: B^{4} \rightarrow E$ is a cross-section, there is a cross-section $f^{\prime}: B^{5} \rightarrow E$ if and only if $\vec{c}^{5}(f)=S q^{2} \gamma$ for some $\gamma \in H^{3}(B)$ when $n=2$, and there is a cross-section $f^{\prime}: B^{6} \rightarrow E$ if and only if $\bar{c}^{6}(f)=S q^{3} \gamma$ for some $\gamma \in H^{3}(B)$ when $n>2$.

This last result agrees with Peterson [15], where he proves that if $n=3$ and $c_{1}(\xi)=c_{2}(\xi)=c_{3}(\xi)=0$, then the third obstruction is

$$
S q_{\phi_{\xi}}^{3}\left(\tilde{c}_{2}\right) \in H^{6}(B) / S q^{3} H^{3}(B),
$$

where $S q_{\phi_{\xi}}^{3}$ is the functional cohomology operation induced by the classifying map $\phi_{\xi}: B \rightarrow B_{U(n)}$ and $S q^{3}$.

8. Principal bundles with fibre $S O(n)$. Bott [5] and Kervaire [11] have calculated $\pi_{i}(S O(n))$ for small values of $i(i \leqq n+4, n \geqq 8)$. For our purposes we will need $\pi_{1}(S O(n))=Z_{2}, \pi_{4}(S O(5))=Z_{2}$, and $\pi_{4 k-1}(S O(n))=Z$, if $n \geqq 4 k+1$.

We will use the following notation for certain cohomology classes of $B_{S O(n)}$.

(i) $\tilde{w}_{i} \in H^{i}\left(B_{S O(n)} ; Z_{2}\right), i=2,3, \cdots, n$, is the $i$ th universal StiefelWhitney class,

(ii) $\tilde{p}_{i} \in H^{4 i}\left(B_{S O(n)}\right), i=1,2, \cdots,[n / 2]$, is the $i$ th universal Pontrjagin class,

(iii) $\tilde{W}_{n} \in H^{n}\left(B_{S O(n)}\right), n$ even, is the universal Euler-Poincaré class.

The classes $\tilde{p}_{i}$ and $\tilde{W}_{n}$ are of infinite order. For further information on the cohomology ring of $B_{S O(n)}$ (and $B_{U(n)}$ ) see Borel [2]. If

$$
\xi=(E, p, B, S O(n), S O(n))
$$

is a principal bundle and $\phi_{\xi}: B \rightarrow B_{S O(n)}$ is a classifying map, then $w_{i}(\xi)$ $=\phi_{\xi}^{*}\left(\tilde{w}_{i}\right), \quad p_{i}(\xi)=\phi_{\xi}^{*}\left(\tilde{p}_{i}\right)$, and $W_{n}(\xi)=\phi_{\xi}^{*}\left(\tilde{W}_{n}\right)$, are the Stiefel-Whitney, Pontrjagin, and Euler-Poincaré classes of $\xi$ respectively.

We propose to calculate the relationship between certain of these classes and the obstructions to cross-sections of the bundle $\xi$. For this purpose we 
will assume that the universal bundle $\xi=\left(E_{S O(n)}, \tilde{p}, B_{S O(n)}, S O(n), S O(n)\right)$ is given a decomposition $\left(\xi, N,\left\{\xi_{m}\right\},\left\{\left(\xi_{m}, 1, \gamma_{m}\right)\right\}\right)$ of length $N \geqq 4[n / 2]$, and that $\tilde{\xi}_{m}=\left(\tilde{E}_{m}, \tilde{p}_{m}, B_{S O(n)}, G_{m}, G_{m}\right)$.

THEOREM 8.1. Let $\lambda^{4 k}(\xi) \in H^{4 k}\left(\tilde{E}_{4 k-2} ; \pi_{4 k-1}(S O(n))\right)=H^{4 k}\left(\tilde{E}_{4 k-2}\right)$, for $n \geqq 4 k+1$, be as previously defined. Then

$$
\tilde{p}_{4 k-2}^{*}\left(\tilde{p}_{k}\right)= \begin{cases} \pm 2(2 k-1) ! \lambda^{4 k}(\xi) & \text { if } k \text { is odd } \\ \pm(2 k-1) ! \lambda^{4 k}(\xi) & \text { if } k \text { is even. }\end{cases}
$$

The proof is similar to that of 7.1 and is omitted.

Corollary 8.2. If $\xi=(E, p, B, S O(n), S O(n))$ is a principal bundle which admits a cross-section $f: B^{4 k-1} \rightarrow E$ for $n>4 k$, then

$$
p_{k}(\xi)= \begin{cases} \pm 2(2 k-1) ! \bar{c}^{4 k}(f) & \text { if } k \text { is odd } \\ \pm(2 k-1) ! \bar{c}^{4 k}(f) & \text { if } k \text { is even } .\end{cases}
$$

The proof is similar to 7.2 .

Corollary 8.3. If $\xi=(E, p, B, S O(n), S O(n))$ is a principal bundle such that $H^{8 k+2}\left(B ; Z_{2}\right)=H^{8 k+1}\left(B ; Z_{2}\right)=0$, the torsion coefficients of $H^{8 k}(B)$ are relatively prime to $(4 k-1)$ !, and the torsion coefficients of $H^{8 k+4}(B)$ are relatively prime to $2(4 k+1)$ !, for $n \geqq 4 k+1$, then $\xi$ admits a cross-section $f: B^{n-1} \rightarrow E$ if and only if $p_{1}(\xi)=p_{2}(\xi)=\cdots=p_{[(n-1) / 4]}(\xi)=0$.

We have not, been able to obtain any relationship between any of the other characteristic classes and higher obstructions at this time.

Before we proceed further, we give the low dimensional integral cohomology of $S O(n)$ and $B_{S O(n)}, n \geqq 5$. For $S O(n)$ we have $H^{1}(S O(n))=0, H^{2}(S O(n))$ $=Z_{2}, H^{3}(S O(n))=Z, H^{4}(S O(n))=Z_{2} ;$ and for $B_{S O(n)}, H^{1}\left(B_{S O(n)}\right)=H^{2}\left(B_{S O(n)}\right)$ $=0, H^{3}\left(B_{S O(n)}\right)=Z_{2}$, and $H^{4}\left(B_{S O(n)}\right)=Z$. The generator of $H^{3}\left(B_{S O(n)}\right)$ is the integral Stiefel-Whitney class $\widetilde{W}_{3}$, and that of $H^{4}\left(B_{S O(n)}\right)$ is the Pontrjagin class $\tilde{p}_{1}$.

LEMma 8.4. If $\dot{\xi}=(E, p, B, S O(n), S O(n))$ is a principal bundle, $n \geqq 5$, if $\phi_{\xi}: B \rightarrow B_{S O(n)}$ is a classifying map, and if $w_{2}(\xi)=\phi_{\xi}^{*}\left(\tilde{w}_{2}\right)=0$, then the generator $u_{3} \in H^{3}(S O(n))$ is transgressive in $\xi$, and if $\tau$ is the transgression, $p_{1}(\xi)$ $= \pm 2 \tau\left(u_{3}\right)$.

Proof. Consider the following portions of the spectral sequences of $\tilde{\xi}$ and $\xi$ and the mapping $\phi_{\xi}^{*}$ between them

$$
\begin{aligned}
& H^{3}(S O(n))=E_{2}^{0,3} \stackrel{\bar{d}_{2}}{\rightarrow} \tilde{E}_{2}^{2,2}=H^{2}\left(B_{S O(n)} ; H^{2}(S O(n))\right) \underset{\phi_{\xi}^{*}}{\stackrel{\alpha}{\rightarrow}} H^{2}\left(B_{S O(n)} ; Z_{2}\right) \\
& \quad \approx \downarrow \\
& H^{3}(S O(n))=E_{2}^{0,3} \stackrel{d_{2}}{\rightarrow} E_{2}^{2,2}=H^{2}\left(B ; H^{2}(S O(n))\right) \stackrel{\alpha}{\approx} \rightarrow H^{2}\left(B ; Z_{2}\right),
\end{aligned}
$$


where $\alpha: H^{2}(S O(n)) \rightarrow Z_{2}$ is a fixed coefficient isomorphism. Since $\phi_{\xi}^{*}\left(\tilde{w}_{2}\right)=0$ and $\tilde{w}_{2}$ generates $H^{2}\left(B_{S O(n)} ; Z_{2}\right), d_{2}: E_{2}^{0,3} \rightarrow E_{2}^{2,2}$ is zero. Since

$$
E_{2}^{3,1}=H^{8}\left(B ; H^{1}(S O(n))\right)=0,
$$

this means that $u_{3} \in H^{3}(S O(n))$ is transgressive in the bundle $\xi$. According to Borel and Hirzebruch [4], in the universal bundle $\xi, 2 u_{3}$ is transgressive and transgresses to $\pm p_{1} \in H^{4}\left(B_{S O(n)}\right)$. From this fact and the fact that $\phi_{\xi}^{*}$ commutes with the transgression, we obtain $p_{1}(\xi)= \pm 2 \tau\left(u_{3}\right)$.

LEMMA 8.5. If $n \geqq 5, k^{4}(S O(n))=0$.

Proof. See Dold and Whitney [7].

By Corollary 6.3, this implies that the secondary obstruction of a principal $S O(n)$-bundle, $n \geqq 5$, is a single cohomology class. This lemma also implies that $G_{3}$ has the homotopy type of $K\left(Z_{2}, 1\right) \times K(Z, 3)$.

ThEOREM 8.6. If $\xi=(E, p, B, S O(n), S O(n))$ is a principal bundle, $n \geqq 5$, and if the Stiefel-Whitney class $w_{2}(\xi) \in H^{2}\left(B ; Z_{2}\right)$ is zero, then the generator $u_{3} \in H^{3}(S O(n))$ is transgressive, and $\tau\left(u_{3}\right)$ is the secondary obstruction to a crosssection, where $\tau$ is the transgression in $\xi$.

Proof. Since $w_{2}(\xi)=0, \xi_{2}=\left(E_{2}, p_{2}, B, G_{2}, G_{2}\right)$ admits a cross-section $f: B \rightarrow E_{2}$. Since $\eta_{3}^{*}: H^{3}\left(G_{3}\right) \rightarrow H^{3}(S O(n))$ is an isomorphism onto, and since transgression commutes with bundle maps, it will be sufficient to prove the theorem in the bundle $\xi_{3}=\left(E_{3}, p_{3}, B, G_{3}, G_{3}\right)$, regarding $u_{3}$ as a generator of $H^{3}\left(G_{3}\right)$. Recall that $G_{2}$ is a $K\left(Z_{2}, 1\right)$ and $G_{3}$ has the homotopy type of $K\left(Z_{2}, 1\right)$ $\times K(Z, 3)$. Thus $H^{3}\left(G_{2}\right)=0$ and $\gamma_{3}^{*}: H^{4}\left(G_{2}\right) \rightarrow H^{4}\left(G_{3}\right)$ is an isomorphism onto, which implies that $\delta^{*}: H^{8}\left(G_{3}\right) \rightarrow H^{4}\left(G_{2}, G_{3}\right)$ is an isomorphism onto. Now consider the commutative diagram

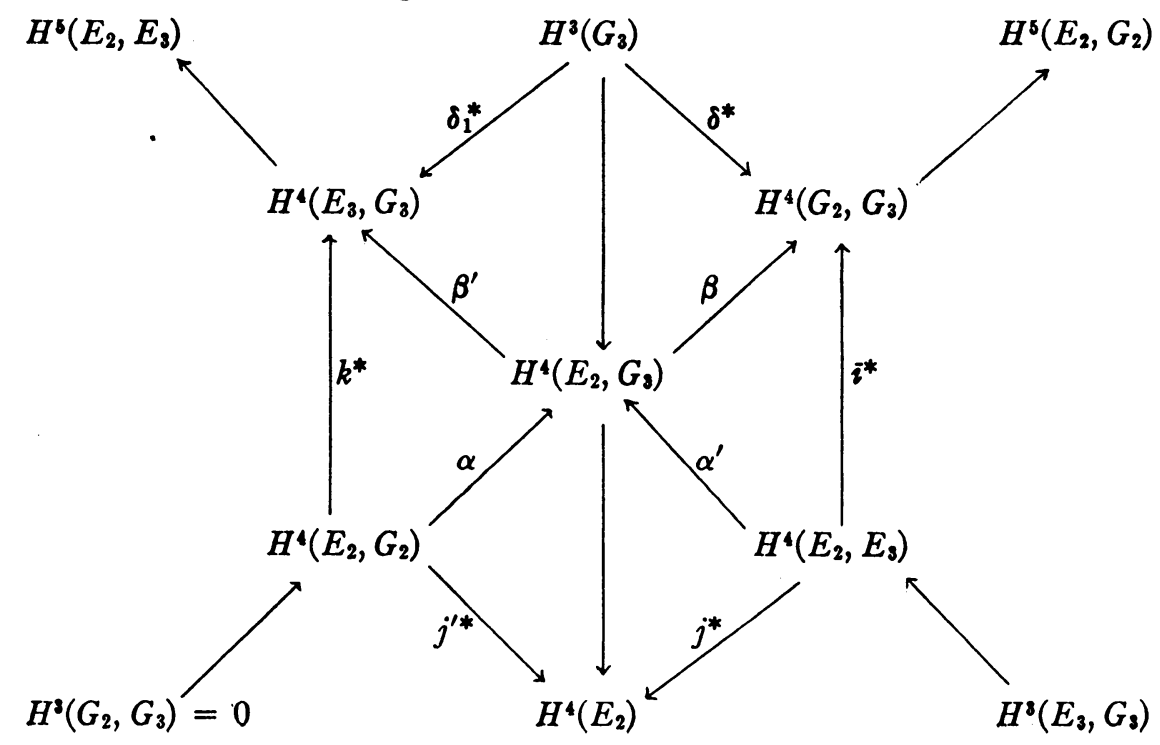


where all sequences through the center term are exact. The homomorphism $k^{*}$ is an isomorphism into, because if $u \in H^{4}\left(E_{2}, G_{2}\right)$ and $k^{*}(u)=0$, then $\beta^{\prime} \alpha(u)=0$, so $\alpha(u)=\alpha^{\prime}(v)$. But $i^{*}(v)=\beta \alpha^{\prime}(v)=\beta \alpha(u)=0$, and since $i^{*}$ is an isomorphism, $v=0$. Thus $0=\alpha^{\prime}(v)=\alpha(u)$, and since $H^{3}\left(G_{2}, G_{3}\right)=0, \alpha$ is an isomorphism into, and $u=0$. This provides an injective direct sum representation $H^{4}\left(E_{2}, G_{3}\right) \approx H^{4}\left(E_{2}, E_{3}\right)+H^{4}\left(E_{2}, G_{2}\right)$, and it is easily verified that $\delta_{1}^{*}\left(u_{3}\right) \in$ Image $k^{*}$. This means that the "hexagonal lemma" of Eilenberg and Steenrod [8, p. 38] applies, and hence

$$
j^{* *} k^{*-1} \delta_{1}^{*}\left(u_{3}\right)=-j^{*} i^{*-1} \delta^{*}\left(u_{3}\right)=\lambda^{4}(\xi),
$$

since we may assume that $\delta^{*}\left(u_{3}\right)=-\theta^{4}\left(G_{2}, G_{3}\right)$, the fundamental class of $\left(G_{2}, G_{3}\right)$.

Now consider the commutative diagram

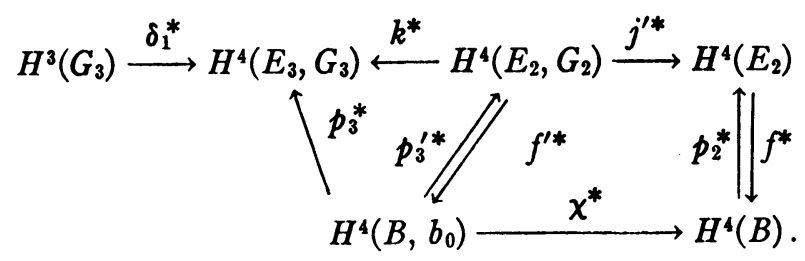

By definition,

$$
\tau\left(u_{3}\right)=\chi^{*} p_{3}^{*-1} \delta_{1}^{*}\left(u_{3}\right)
$$

so

$$
p_{2}^{*} \tau\left(u_{3}\right)=p_{2}^{*} \chi^{*} p_{3}^{*-1} \delta_{1}^{*}\left(u_{3}\right)=j^{*} k^{*-1} \delta_{1}^{*}\left(u_{3}\right)=\lambda^{4}(\xi),
$$

and

$$
\tau\left(u_{3}\right)=f^{*} p_{2}^{*} \tau\left(u_{3}\right)=f^{*}\left(\lambda^{4}(\xi)\right)=\bar{c}^{4}(f) .
$$

In the following lemma, $\theta_{3}$ denotes the 3-dimensional generator of $H^{3}\left(G_{i}\right)=Z$ for $i=3,4,6$, and $\theta_{1}$ denotes the 1-dimensional generator of $H^{1}\left(G_{i} ; Z_{2}\right)$ for $i=3,4,6$. The sevenfold cup-product of $\theta_{1}$ with itself will be denoted by $\theta_{1}^{7}$. In part (iv), the coefficients are in the group $\pi_{7}(S O(8))=Z+Z$, and the primes are used to distinguish the elements of the two summands of $H^{8}\left(G_{6} ; Z+Z\right)$ determined by a decomposition of the coefficient group.

LEMMA 8.7. (i) $k^{5}(S O(5))=S q^{2} \theta_{3} \in H^{5}\left(G_{3} ; \pi_{4}(S O(5))\right)=H^{5}\left(G_{3} ; Z_{2}\right)$,

(ii) $k^{6}(S O(6))=S q^{3} \theta_{3} \in H^{6}\left(G_{4} ; \pi_{5}(S O(6))\right)=H^{6}\left(G_{4} ; Z\right)$,

(iii) $k^{8}(S O(7))=\Delta_{3} \odot_{3}^{1} \theta_{3}+\Delta_{2} \theta_{1}^{7} \in H^{8}\left(G_{6} ; \pi_{7}(S O(7))\right)=H^{8}\left(G_{6} ; Z\right)$,

(iv) $k^{8}(S O(8))=\Delta_{3} \odot_{3}^{1} \theta_{3}+\Delta_{3} \rho_{3} \theta_{3}^{\prime}+\Delta_{2} \theta_{1}^{7}+\Delta_{2} \theta_{1}^{\prime 7} \in H^{8}\left(G_{6} ; \pi_{7}(S O(8))\right)=H^{8}\left(G_{6}\right.$; $Z+Z)$,

(v) $k^{8}(S O(n))=\Delta_{3} \mathcal{P}_{3}^{1} \theta_{3} \in H^{8}\left(G_{6} ; \pi_{7}(S O(n))\right)=H^{8}\left(G_{6} ; Z\right), n \geqq 9$, where $\Delta_{n}$ is the Bockstein operator associated with the coefficient sequence $0 \rightarrow Z \rightarrow Z \rightarrow Z_{n} \rightarrow 0$, and $\mathcal{P}_{3}^{1}$ is the Steenrod reduced cube. 
The proof is similar to the proof of 7.5 , but is much more complicated. We omit the proof.

Proposition 8.8. Let $\xi=(E, p, B, S O(n), S O(n))$ be a principal bundle which admits a cross-section $f_{0}: B^{4} \rightarrow E$, where $n \geqq 5$. Then if $d \in H^{1}\left(B ; Z_{2}\right)$ and $\gamma \in H^{3}(B ; Z)$, there is a cross-section $f_{1}: B^{4} \rightarrow E$ such that $\bar{d}\left(f_{0}, f_{1}\right)=d$ and $\bar{\gamma}^{3}\left(f_{0}, f_{1}\right)=\gamma$, where $\bar{\gamma}^{3}\left(f_{0}, f_{1}\right)=h_{f_{0} \gamma_{1}}^{*}\left(u_{3}\right)$ and $u_{3}$ is a generator of $H^{3}(S O(n))$.

The proof is similar to the proof of 7.6.

THEOREM 8.9. Let $\xi=(E, p, B, S O(n), S O(n))$ be a principal bundle, where $n \geqq 5$. Then the following are true.

(i) The primary obstruction to a cross-section is $w_{2}(\xi) \in H^{2}\left(B ; Z_{2}\right)$.

(ii) If $w_{2}(\xi)=0$, the secondary obstruction to a cross-section is the transgression of a generator $u_{3} \in H^{3}(S O(n))$, and if $O^{4} \in H^{4}(B ; Z)$ denotes the obstruction, then $p_{1}(\xi)= \pm 2 O^{4}$.

(iii) Let $f: B^{4} \rightarrow E$ be a cross-section of $\xi$, i.e., the first two obstructions vanish. Then

if the fibre is $S O(5), \xi$ admits a cross-section over the 5-skeleton if and only if $\bar{c}^{5}(f)=S q^{2} \gamma$ for some $\gamma \in H^{3}(B ; Z)$,

if the fibre is $S O(6), \xi$ admits a cross-section over the 6-skeleton if and only if $\bar{c}^{6}(f)=S q^{3} \gamma$ for some $\gamma \in H^{3}(B ; Z)$,

if the fibre is $S O(7), \xi$ admits a cross-section over the 8-skeleton if and only if $\bar{c}^{8}(f)=\Delta_{3} \odot_{3}^{1} \gamma+\Delta_{2} d^{7}$ for some $\gamma \in H^{3}(B ; Z)$ and $d \in H^{1}\left(B ; Z_{2}\right)$,

if the fibre is $S O(8), \xi$ admits a cross-section over the 8-skeleton if and only if $\bar{c}^{8}(f)=\Delta_{3} \rho_{3}^{1} \gamma+\Delta_{3} \rho_{3}^{1} \gamma^{\prime}+\Delta_{2} d^{7}+\Delta_{2} d^{\prime 7}$ for some $\gamma, \gamma^{\prime} \in H^{3}(B)$ and $d, d^{\prime} \in H^{1}\left(B ; Z_{2}\right)$,

if the fibre is $S O(n), n \geqq 9, \xi$ admits a cross-section over the 8-skeleton if and only if $\bar{c}^{8}(f)=\Delta_{3} \odot_{3}^{1} \gamma$ for some $\gamma \in H^{3}(B ; Z)$.

Part (iii) is an application of 6.6 and Lemma 8.8.

9. Principal bundles with fibre $S p(n)$. Bott [5] has proved that for $i \leqq 4 n+1, \pi_{i}(S p(n))=Z$ if $i \equiv 3(\bmod 4), \pi_{i}(S p(n))=Z_{2}$ if $i \equiv 4,5(\bmod 8)$, and $\pi_{i}(S p(n))=0$ if $i \equiv 0,1,2,6(\bmod 8)$. In particular, $\pi_{i}(S p(n))=0$ for $i<3, \pi_{3}(S p(n))=Z$, and $\pi_{4}(S p(n))=Z_{2}$.

We will use $\tilde{e}_{i} \in H^{4 i}\left(B_{S p(n)} ; Z\right)$ to denote the symplectic Pontrjagin class [3] of the universal $S p(n)$-bundle $\xi$, and if $\xi=(E, p, B, S p(n), S p(n))$ is a bundle with classifying map $\phi_{\xi}: B \rightarrow B_{S p(n)}, e_{i}(\xi)=\phi_{\xi}^{*}\left(\tilde{e}_{i}\right)$ will denote the symplectic Pontrjagin class of $\xi$. We will assume that we have a decomposition $\left(\xi, N,\left\{\tilde{\xi}_{m}\right\},\left\{\left(\tilde{g}_{m}, 1, \gamma_{m}\right)\right\}\right)$ of the universal bundle $\xi$ which is of length $N \geqq 4 n$.

THEOREM 9.1. Let $\lambda^{4 k}(\tilde{\xi}) \in H^{4 k}\left(\tilde{E}_{4 k-2} ; \pi_{4 k-1}(S p(n))\right)=H^{4 k}\left(\tilde{E}_{4 k-2} ; Z\right)$ for $k \leqq n$, be as previously defined. Then

$$
\tilde{p}_{4 k-2}^{*}\left(\tilde{e}_{k}\right)= \begin{cases} \pm(2 k-1) ! \lambda^{4 k}(\xi) & \text { if } k \text { is odd } \\ \pm 2(2 k-1) ! \lambda^{4 k}(\xi) & \text { if } k \text { is even }\end{cases}
$$


The proof is similar to the proof of 7.1.

Corollary 9.2. If $\xi=(E, p, B, S p(n), S p(n))$ is a principal bundle which admits a cross-section $f: B^{4 k-1} \rightarrow E$, where $k \leqq n$, then

$$
e_{k}(\xi)= \begin{cases} \pm(2 k-1) ! \bar{c}^{4 k}(f) & \text { if } k \text { is odd } \\ \pm 2(2 k-1) ! \bar{c}^{4 k}(f) & \text { if } k \text { is even }\end{cases}
$$

The proof is similar to the proof of 7.2.

COROLlary 9.3. If $M^{4 n}$ is an almost quaternionic (4n)-manifold such that $H^{i}\left(M^{4 n} ; Z_{2}\right)=0$ for $i \equiv 5,6(\bmod 8)$ and such that the cohomology torsion coefficients in dimension $8 i$ are relatively prime to $2(4 i-1)$ ! and in dimension $8 i+4$ are relatively prime to $(4 i+1)$ !, then $M^{4 n}$ is parallelizable if and only if $e_{1}(\xi)=e_{2}(\xi)=\cdots=e_{n}(\xi)=0$, where $e_{i}(\xi)$ is the Pontrjagin class of the tangent bundle $\xi$ of $M^{4 n}$ :

LEMmA 9.4. The invariant $k^{5}(S p(n))=S q^{2} \theta_{3} \in H^{5}\left(G_{3} ; Z_{2}\right)$, where $\theta_{3}$ is the fundamental class of $G_{3}=K(Z, 3)$.

The proof is very easy.

Theorem 9.5. If $\xi=(E, p, B, S p(n), S p(n))$ is a principal bundle, then

(i) the primary obstruction to a cross-section is $e_{1}(\xi)$,

(ii) if $e_{1}(\xi)=0$ and $f: B^{4} \rightarrow E$ is a cross-section, then there is a cross-section $f^{\prime}: B^{5} \rightarrow E$ if and only if there is an element $d \in H^{3}(B ; Z)$ such that $\bar{c}^{5}(f)=S q^{2} d$.

Proof. Part (i) follows from 9.2, and (ii) follows from Corollary 6.6 when we take account of 9.4 .

\section{BiBLIOGRAPHY}

1. A. L. Blakers and W. S. Massey, The homotopy groups of a triad. I, Ann. of Math. vol. 53 (1951) pp. 161-205.

2. A. Borel, Selected topics in the homology theory of fibre bundles, Mimeographed lecture notes, University of Chicago, 1954.

3. A. Borel and F. Hirzebruch. Characteristic classes and homogeneous spaces. I, Amer. J. Math. vol. 80 (1958) pp. 458-538.

4. Characteristic classes and homogeneous spaces. II, Amer. J. Math. vol. 81 (1959) pp. 315-381.

5. R. Bott, The stable homotopy of the classical groups, Proc. Nat. Acad. Sci. U.S.A. vol. 43 (1957) pp. 933-935.

6. H. Cartan, Séminaire de topologie algebrique, Paris, Ecole Normal Supérieure, 1949-1950.

7. A. Dold and H. Whitney, Classification of oriented sphere bundles over a 4-complex, Ann. of Math. vol. 69 (1959) pp. 667-677.

8. S. Eilenberg and N. Steenrod, Foundations of algebraic topology, Princeton University Press, 1952.

9. R. Hermann, Secondary obstructions for fibre spaces, Bull. Amer. Math. Soc. vol. 65 (1959) pp. 5-8. 
10. M. Kervaire, On the Pontrjagin classes of certain $S O(n)$-bundles over manifolds, Amer. J. Math. vol. 80 (1958) pp. 632-638.

11. - Some non-stable homotopy groups of Lie groups, Illinois J. Math. vol. 4 (1960) pp. 161-169.

12. S. D. Liao, On the theory of obstructions of fibre bundles, Ann. of Math. vol. 60 (1954) pp. 146-191.

13. J. Milnor, Construction of universal bundles. I, Ann. of Math. vol. 63(1956) pp. 272-284.

14. - Construction of universal bundles. II, Ann. of Math. vol. 63 (1956) pp. 430-436.

15. F. Peterson, Some remarks on Chern classes, Ann. of Math. vol. 69 (1959) pp. 414-419.

16. N. Steenrod, The topology of fibre bundles, Princeton University Press, 1951.

17. J. H. C. Whitehead, Combinatorial homotopy. I, Bull. Amer. Math. Soc. vol. 55 (1949) pp. 213-245.

Brown UNIVERSITY,

Providence, Rhode Island 